\title{
XXI.
}

Aus dem pharmakologischen Institut zu Heidelberg.

\section{Ueber Diurese.}

II. Mittheilung: Vergleich der diuretischen Wirksamkeit isotonischer Salzlösungen.

Von

Dr. R. Magnus, Assistenten des Instituts.

(Mit 8 Curven im Text.)

\section{Einleitung.}

Wie durch die Arbeiten von v. Limbeck1), Heidenhain ²), $M u ̈ n z e r^{3}$ ) festgestellt worden ist, rufen Lösungen verschiedener Salze von gleichem Procentgehalt bei intravenöser Infusion nicht die gleiche Diurese hervor. Diese erreicht um so höhere Werthe, je niedriger das Moleculargewieht des betreffenden Salzes ist. Es nimmt also mit steigender molecularer Concentration ${ }^{4}$ ) der Infusionsflusssigkeit ihre diuretische Wirkung zu. Sicher gilt dieses Gesetz für die Natronsalze einbasischer Säuren (Munzer). Das Gleiche haben kürzlich Hédon und Arrous ${ }^{5}$ ) für die verschiedenen Zuckerarten gezeigt. In 25 procent. Lösung wirkten die Zuckerarten mit grossem Molekül viel weniger harntreibend als Pentosen und Hexosen.

Erweist sich also die Diurese abhängig von „ colligativen" Eigenschaften der betreffenden Salzlösungen (molekularer Concentration, Wasseranziehungsvermögen, osmotiseher Druek), so entstehen zwei Fragen:

1. Wie ist dieser Zusammenhang zu erklären?

2. Ist die molekulare Concentration der einzige Factor, von dem die diuretische Kraft einer Salzlösung abhängt?

1) v. Limbeck, Archiv f. experiment. Pathol. u. Pharmakol. Bd. XXV. S. 86.1888 .

2) Heidenhain, Pflüger's Archiv Bu. IL. S. 269. 1891.

3) Münzer, Archiv f, experiment. Pathol. u. Pharmakol. Bd. XLI. S. 74. 1898.

4) Wenn im Folgenden von gleicher molekularer Concentration die Rede ist, so ist stets die Ionisation mit berücksichtigt. Es sind also isotonische Lösungen gemeint.

5) Hédon und Arrous, C. R. Soc. Biol. 1899, S. 879. 
Was den ersten Punkt anbelangt, so habe ich in einer kürzlich erschienenen Arbeit ${ }^{1}$ ) gezeigt, dass man hochgradige Diuresen erzeugen kann, wenn allein der Wassergehalt des Blutes gesteigert, dagegen der Salzgehalt and der osmotische Druck vermindert ist. Im Anschluss daran war die Frage discutirt, ob die diuretische Wirksamkeit starker Salzlösungen nicht darauf beruhe, dass sie, wie zuerst $\mathrm{von} \mathrm{Brasol}^{2}$ ) und $\mathrm{Klikowicz}{ }^{3}$ ) nachgewiesen haben, einen Strom von Wasser aus den Geweben ins Blut hervorrufen. Dadurch würde der Niere ein wasserreicheres Blut geboten und auf die Blutverdünnung, nicht aber anf die Salzvermehrung antworte die Niere mit Steigerung der Harnmenge. Diese Ansicht war schon früher von Starling ${ }^{4}$ ) und Cohnstein ${ }^{5}$ ) vertreten. Sie erklärt hinreichend, warum mit Steigerung des wasseranziehenden Vermögens der Salzlösung ihre diuretische Wirkung steigt. Je stärker das wasseranziehende Vermögen der Salzlösung, desto mehr Wasser wird ceteris paribus in die Blutbahn gezogen, desto höher muss die Harnfluth schwellen ${ }^{6}$ ). Gleichzeitig hatte ich aber auf einige Schwierigkeiten hingewiesen, die dieser Ansicht dadurch erwachsen, dass gegen Ende einer Salzdiurese der Parallelismus zwischen Blutverdünnung und Harnfluth aufhört, indem bald bei noch bestehender Blutverdünnung die Diurese erlischt, bald die Diurese noch andauert, obwohl keine Blutverdünnung, manchmal sogar eine Bluteindickung besteht.

Eine experimentelle Untersuchung dieser Fragen ist das Thema vorliegender Arbeit. Ist die Anschauung richtig, dass Salzlösungen nur deshalb diuretisch wirken, weil sie Blutverdännung hervorrufen, so müssen Lösungen, welche gleiche Bl utverd tinn ung erzeugen, auch gleiche Diurese bewirken.

Um möglichst günstige Bedingungen für den Eintritt gleicher Blutverdünnung bei Anwendung versehiedener Salze zu schaffen, wurden den Versuchsthieren gleichlange relativ gleiche Mengen miteinander isotonischer Lösungen der zu vergleichenden Salze intravenös eingeführt. Dabei waren folgende Resultate möglich:

1) Archiv f. experiment. Pathol. u. Pharmakol. Bd. XLIV. S. 101. 1900. Im Folgenden als ,I. Mittheilung“ bezeichnet.

2) von Brasol, Archiv f. (Anat.) u. Physiol. 1884. S. 210.

3) Klikowicz, Ebenda 1886. S. 518.

4) Starling, Journal of Physiology Vol. XXIV. S. 317. 1899.

5) Cohnstein, Pfiuger's Archiv Bd. LXII. S. 73. 1895.

6) Auf die event. verschiedene Durchgăngigkeit der Capillarwände für die einzelnen Salze ist hierbei zunächst keine Rücksicht genommen. 
1. Die verglichenen Salzlösungen bewirken gleichgrosse Diuresen.

2. Sie rufen verschiedene Harnfluth hervor. Dies kann bedingt sein a) dadurch, dass auch die Blutverdinnnung eine verschiedene ist, b) oder die Blutverdünnung ist die gleiche, die Diurese aber trotzdem rerschieden gross.

Eine Untersuchung in der angegebenen Richtung liess noch die Beantwortung der zweiten obigen Frage erwarten. Ist nämlich die molekulare Concentration der Einlaufsflüssigkeit nicht der einzige Factor, von dem die Grösse der erzeugten Diurese abhängt, so mussten diese anderen mitbestimmenden Einflüsse dann am deutlichsten hervortreten, wenn man die Salzwirkungen unter den sonst gleichbleibenden Bedingungen isotonischer Lösungen untersuchte. So war zu hoffen, dass sich ein besserer Einblick als bisher in das Zustandekommen der Diurese gewinnen liess.

Ausfïhrliche Untersuchungen, die sich mit der diuretischen Wirksamkeit unter sich isotonischer Salzlösungen befassen, liegen bislang nicht vor. v. Limbeck beribrt diesen Punkt nur kurz am Ende der erwähnten Abhandlung. Er stellte sich nach $\mathrm{Hamburger's} \mathrm{Blut-}$ körperchenmethode Salzlösungen dar, die dem Blute isotonisch waren. Er sagt: „Es hat sich herausgestellt, dass intravenöse Injection isotonischer Lösung der verschiedensten Salze eine sehr geringe, nahezu gleiche Harnausscheidung einleiteten (dies gilt von Bromid, Jodid, Sulfat, Nitrat, Chlorat, Acetat, Phosphat und Tartrat), dass nur das Chlorid, entsprechend seiner Eigenschaft als physiologisches Salz, $\chi \alpha \tau^{3}{ }^{3} \xi \circ \chi \chi \dot{\eta} \nu$ eine auffällige Steigerung der Secretion anslöste." Nähere Angaben über diese Versuche, die nur als vorläufige bezeichnet werden, fehlen. - Ferner geben Hédon und Arrous an, dass 25 procent. Lösungen isomerer Zuckerarten, die also isotonisch sind, nicht genau die gleiche Diurese hervorriefen. Sie führen diese Erscheinung darauf zurtick, dass von den verschiedenen Zuckern im Körper während der Versuchszeit nicht die gleichen Mengen zerstört würden. Weitere Angaben konnte ich in der Litteratur nicht auffinden.

\section{Versuche.}

Es erschien von Vortheil, nicht eine grosse Menge der verschiedensten Salze vergleichend zu untersuchen, sondern nur zwei einander gegenüber zu stellen, diese dafür aber an zahlreichen Versuchsthieren und von verschiedenen Gesichtspunkten aus zu verwerthen. Gewählt wurden hierzu das Kochsalz und das Glaubersalz, für welche beide v. Limbeck und Münzer starke diuretische Wirkungen festgestellt hatten. Für NaCl lag die Angabe v. Limbeck's 
vor, dass es am stärksten von allen Salzen wirke, was auch Münzer bestätigt hat. Mit dem Kochsalz gerade das Glaubersalz zu vergleichen, dazu veranlasste die merkwürdige Angabe Mün zer's, dass $\mathrm{Na}_{2} \mathrm{SO}_{4}$ zwar weniger diuretisch wie $\mathrm{NaCl}$ wirke, statt dessen aber das harnfähigere sei, da von der eingeführten Salzmenge ein grösserer Bruchtheil bei Glaubersalı als bei Kochsalz in den Harn gehe. Weiter schienen $\mathrm{NaCl}$ und $\mathrm{Na}_{2} \mathrm{SO}_{4}$ am ersten Unterschiede zu versprechen, da durch die Untersuchungen über ihre Resorbirbarkeit im Darm festgestellt ist, dass sie sich wenigstens hierbei stark verschieden verhalten.

Als Einlaufsflüssigkeit diente einerseits eine Kochsalzlösung von 4,90 Proc., deren Gehalt durch Titration bestimmt wurde. Nach dieser wurde eine ihr isotonische Glaubersalzlösung hergestellt. Hierzu wurde jedoch nicht die Methode der Gefrierpunktserniedrigung, bezw. der Beckmann'sche Apparat benutzt. Mittels desselben hätte man 2 Lösungen erbalten, die im Glase isotonisch sind, jedoch, wenn sie langsam ins Blut fliessen, dort stark verdünnt werden, dabei verschieden starke Dissociation erleiden und alsbald von einander verschieden werden. Vielmehr schien es richtiger, die Isotonie bei grösserer Verdünnung festzustellen. Dazu ist die Methode $\mathrm{Ham}$ burger's geeignet. Diese bestimmt die Isotonie dureh Ermittelung der Concentration, bei der gerade der rothe Blutfarbstoff aus zugesetzten Erythrocyten austritt. Erstens wird hierbei die gleiche osmotische Spannung für Verdünnungen ermittelt, welche denen sehr viel näher kommen, die die betreffenden Infusionsflüssigkeiten im Körper erleiden, und zweitens dienen als Reagens thierisehe Zellen, so dass gleich nach diesen die lsotonie bestimmt werden konnte. Es fand sich, dass nach Hamburger's Methode unter Benutzung von Ochsenblut einer 4,90 procent. NaCl-Lösung isotonisch war eine $\mathrm{Na}_{2} \mathrm{SO}_{4}$-Lösung von

$$
\begin{aligned}
& 7,853 \\
& \text { im Mittel: } 7,851 \\
& \hline \text { 7,852 Proc., auf wasserfreies Salz be- }
\end{aligned}
$$
rechnet. Beide Lösungen wurden vor Beginn der Versuche in genügender Menge hergestellt, so dass der erste Vorrath für alle Experimente ausreichte, und die Flüssigkeiten in Flaschen mit gut eingeschliffenen Stopfen aufbewahrt.

A. Vergleichend diuretische Versuche.

Diese erste Versuchsreihe bezweckte an einer Anzahl von Kaninchen festzustellen, ob überhaupt Unterschiede in der diuretischen 
Wirksamkeit der beiden Salzlösungen vorhanden sind. Gleichheit der Versuchsbedingung schien hier erstes Erforderniss. Deshalb wurden die Thiere nach dem Vorschlage v. Limbeck's auf möglichst gleichen und niedrigen Wassergehalt gebracht, indem jedes in einem besonderen Käfig isolirt wurde, zweimal 24 Stunden hungerte und durstete, am dritten und vierten Tage je $30 \mathrm{~g}$ pro Kilogramm trocknen Hafers ohne Wasser erbielten und am fünften Tage zum Versuch benutzt wurden. Hierdurch wird, wie der genannte Autor ausführte, ein wenigstens annähernd gleicher Wasserbestand des Körpers erzielt. Und nur unter dieser Vorbedingung lassen sich überhaupt Vergleiche zwischen verschiedenen Thieren anstellen. In der That gelingt es auf diese Weise, die normale Harnausscheidung so herabzudrücken, dass nur 0-11/2 Tropfen in 10 Minuten secernirt werden. Auf diese niedrigen Werthe setzt sich die später eingeleitete Diurese mit deutlich abgesetzten, verhältnissmässig constanten Werthen auf.

Dem so vorbereiteten Kaninchen wurden dann Glaskanülen in beide Ureteren gebunden, aus denen der Harn in ein vorgelegtes Schälchen tropfte, welches, um Verdunstung zu vermeiden, mit einem Uhrglas bedeckt war. Die Normalsecretion wurde mindestens $10 \mathrm{Mi}$ nuten lang bestimmt. Sie hielt sich stets in den oben angegebenen niedrigen Werthen. Darauf lief in allen Versuchen genau 50 Minuten lang eine der beiden Salzlösungen bei Körpertemperatur in die Vena jugularis ein, mit einer Einlanfsgeschwindigkeit von $0,5 \mathrm{~cm}$ pro Minute und Kilogramm des Thieres, so dass im ganzen $25 \mathrm{cem}$ pro Kilogramm infundirt wurden. Die Geschwindigkeit wurde durch eine Schraubklemme regulirt und auf Constanz der Einlaufsmenge und-Geschwindigkeit besondere Sorgfalt verwandt. Inzwisehen war der producirte Harn von 10 zu 10 Minuten gemessen und der Verlauf der Diurese noch 3 Stunden lang nach Beendigung des Einlaufs weiter verfolgt. Der Urin wurde in 2 Portionen vereinigt, die erste enthielt die während der Infusion, die zweite die nachher secernirte Menge. Hierin wurde später der $\mathrm{NaCl}$, bezw. $\mathrm{Na}_{2} \mathrm{SO}_{4}$-Gehalt bestimmt. Vor Beginn des Einlaufs, am Schluss der Infusion und bei Beendigung des ganzes Versuches wurde je ein Blutstropfen aus der Carotis entnommen und für die Hämometerbestimmung nach Miesch er benütgt (vgl. unten S. 408).

Der Erfolg der Infusion war der, dass in allen Fällen eine dentliche Diurese auftrat. Dieselbe stieg während des Einlaufs an und ging nach dem Aufhören desselben anfangs schnell, später allmählicher zurück. Nach 4 Stunden (der Versuchsdauer) war sie bis oder fast bis zur Norm zurückgekehrt. 
Die folgende Tabelle giebt an, wieviel in jedem einzelnen Versuche an Wasser und Salz ausgeschieden wurde, und zwar im Procent der eingelaufenen Menge. Da pro Kilogramm Thier stets das gleiche Quantum einlief, so sind diese Diuresenwerthe direct vergleichbar. In den ersten beiden Spalten ist die Ausscheidung während des Einlaufes (also der ersten 50 Minuten), in den letzten beiden die Ausscheidung während des ganzen Versuches angegeben.

TABelle I.

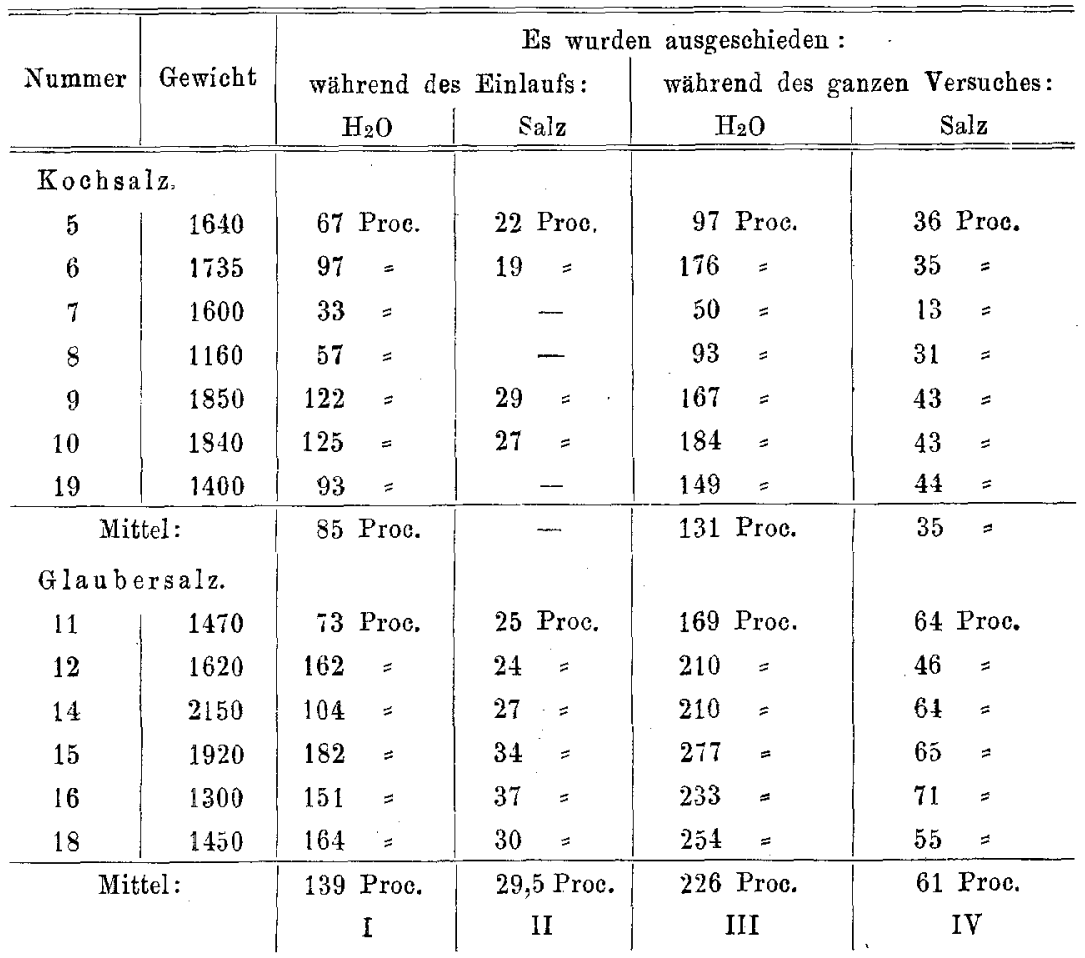

Das Resultat ist unzweideutig. Lässt man bei Kaninchen in gleicher Zeit gleiche Mengen isotonischer Lösungen von Kochsalz und Glaubersalz einlaufen, so erhält man nach Glaubersalz bedeutend stärkere Diuresen, als nach Kochsalz. Unter den oben geschilderten Versuchsbedingungen waren nach Schluss des Einlaufs ausgeschieden in den Kochsalzversuchen 33-125 Proc., im Mittel 85 Proc. des Einlaufswassers und 19-29 Proc. des eingeführten Salzes. In den Glaubersalzversuchen 73-182 Proc., im Mittel 139 Proc. des Einlaufswassers und 24-37 Proc., im Mittel 29,5 Proc. des eingeführten Salzes. Im Verlauf des ganzen Versuches (4 Stunden) wurden in 
den Kochsalzversuchen 50-184 Proc., im Mittel 131 Proc. des Wassers und 13-44 Proc., im Mittel 35 Proc. des Salzes; in den Glanbersalzversuchen 169-277 Proc., im Mittel 226 Proc. des Wassers, und 46-71 Proe., im Mittel 61 Proc. des Salzes ausgeschieden. Die Glaubersalzdiurese ist also fast doppelt so stark, wie die Kochsalzdiurese. Man kann dabei nicht sagen, dass das Kochsalz diuretischer wirke, während das Glaubersalz harnfähiger sei. Vielmehr ist Glaubersalz sowohl harnfähiger, als a uch diuretisch wirksamer. Diese Unterschiede werden bei näherer Betrachtung noch dentlicher. Bei Schluss der Infusion (Col. I) ist in den Kochsalzversuchen bis auf 2 Fälle (9 und 10) noch nicht so viel Wasser ausgeschieden, wie eingelaufen ist. Dagegen beträgt bis zu diesem Zeitpunkt die Glaubersalzdiurese mit Ausnahme eines Versuches (11) schon mehr als 100 Proc. der Einlanfsmenge. Ja, in einzelnen Kochsalzversuchen $(5,7$ und 8 ) blieb die wälrend des ganzen Versuches ausgeschiedene Harnmenge hinter der Einlaufmenge zurück. Auf der anderen Seite sieht man, dass in den $\mathrm{Na}_{2} \mathrm{SO}_{4}$-Versuchen die Gesammtharnmenge in 5 von 6 Fällen mehr als das doppelte des infundirten betrug.

Zwischen der Menge des ausgeschiedenen Salzes (35 und 61 Proc.) und Wassers (131 und 226 Proc. lassen die Mittelzahlen interessante Beziehungen erkennen. Es ist nämlich in beiden Fällen das Verhältniss von Salz und Wasser ungefähr das Gleiche:

$$
131: 35=226: 60,4 \text { (statt } 61 \text { ). }
$$

Das heisst: Bei Einfuhr isotonischer Lösungen scheidet die Niere Salz und Wasser in ungefähr gleichem Verhältniss ab, je mehr Salz, desto mehr Wasser. Je harnfähiger ein Salz ist, desto mehr Wasser wird zugleich mit ihm ausg'eschieden. Dasselbe Resultat ergiebt sich, wenn man die Concentrationen der Harne an den betreffenden Salzen miteinander vergleicht.

Die Kochsalzconcentrationen waren:

$\begin{array}{llllllll}\text { Versuchs-Nr. } & 5 & 6 & 7 & 8 & 9 & 10 & 19\end{array}$

Concentration $1,84 \quad 1,04 \quad 1,251,63 \quad 1,26 \quad 1,131,43$

Im Mittel 1,37 Proc. ${ }^{1}$ )

Die Glaubersalzconcentrationen:

$\begin{array}{ccccccc}\text { Versuchs-Nr. } & 11 & 12 & 14 & 15 & 16 & 18 \\ \text { Concentration } & 2,98 & 1,73 & 2,38, & 1,83 & 2,39 & 1,72 \\ \text { Im Mittel } & \left.2,17 \text { Proc. }{ }^{1}\right) .\end{array}$

1) Der höchste Procentgehalt einer Einzelportion an Kochsalz betrug 2,40 Proc. (Versuch 5), an Glaubersalz 3,27 Proc. (Versuch 16), also noch höhere Werthe, als Münzer (a. a. O. S. 89) fand. 
Vergleich der diuretischen Wirksamkeit isotonischer Salzlösungen. 403

Nun zeigt sich, dass einer Kochsalzlösung von 1,37 Proc. eine Glaubersalzlösung von 2,17 Proc. ungefälr isotonisch ist. (Nach meinen Bestimmungen müsste die $\mathrm{Na}_{2} \mathrm{SO}_{4}$-Lösung 2,17 Proc., nach den Köppe's 1) 2,16 Proc. enthalten).

Es kann natürlich nur ein Zufall sein, dass diese Mittelzahlen aus je 6 bezw. 7 Versuchen so ausgezeichnet zu den angeführten Proportionen stimmen. Jedenfalls deuten sie aber in der angegebenen Richtung. Diese Abhängigkeit der Harnmenge von der ausgeschiedenen Salzmenge bei Infusion isotonischer Lösungen würde ein sehr interessantes Licht auf die Vorgänge bei der Diurese werfen. Doch kann man sie nur als allgemeine Regel aufstellen. In den Einzelversuchen treten immerhin deutliche Abweichungen bis zu 10 Proc. nach oben oder unten auf. Das lehren aber jedenfalls die Mittelzahlen, dass es nicht angängig ist, bei der Salzdiurese die Ausscheidung der Wassermenge unabhängig von den ansgeschiedenen Salzmengen zu betrachten, sondern dass beide in einer gewissen Beziehung zu einander stehen. Welcher Art diese sind, darauf kann erst am Schlusse dieser Arbeit eingegangen werden.

Was den Verlauf der Diurese nach beiden Salzen anbetrifft, so steigt die Glaubersalzdiurese rascher an, erreicht manchmal schon vor Ende des Einlaufs ihren höchsten Werth und sinkt nach Schluss der Infusion langsamer ab als die Koohsalzdiurese. Diese erhebt sich erst später, erreicht ihre höchsten Werthe erst am Schlusse des Einlaufs und sinkt darnach oft rapide $a b$. So wird die Kochsalzcurve viel spitzer, als die für Glaubersalz. Als Beispiel dienen hier zwei Curven von Versuch $9(\mathrm{NaCl})$ und $15\left(\mathrm{Na}_{2} \mathrm{SO}_{4}\right)$, welche diese Untersehiede gut hervortreten lassen, weil bei ihnen die maximale Ausscheidung in den letzten 10 Minuten des Einlaufs, pro Körperkilo berechnet, ungefähr die gleiche war. Die Gesammtausscheidung betrug in dem ersten Falle 167, im zweiten 277 Proc. der Einlaufsmenge. (S. Curve 1 anf folgender Seite.)

Der Harn war in allen Fällen zuckerhaltig mit Ausnahme von Versuch 8. Es ist dieses die von Bock u. Hoffmann ${ }^{2}$ ) beschriebene Glykosurie nach Infusion von Salzlösungen. Eiweiss fand sich manchmal in geringen Spuren, vermuthlich immer bedingt durch geringe Blutbeimengungen, welche sich nach Einführung von Ureterenkaniulen nicht stets vermeiden lassen. Der Allgemeinzustand der Thiere war während der Dauer der Versuche ein guter. Nach grossen Wasserverlusten durch die Diurese liess sich die von Münzer (a. a. 0.),

1) Köppe, Archiv f. (Anat. u.) Physiol. 1895. S. 171.

2) Bock und Hoffmann, Archiv f. Anat. u. Physiol. 187L, S. 550. 
beschriebene Steigerung der Reflexerregbarkeit bemerken. Doch kam es nie zu Krampfanfällen. In allen Versuchen war die Niere das einzige Organ der Wasserausscheidung. Nur in einem Versuche (Nr.9 Kochsalzinfusion) trat 20 Minuten nach Schluss des Einlaufs diarrhöische Stuhlentleerung auf. Speichelfuss stellte sich bei keinem Thiere ein.

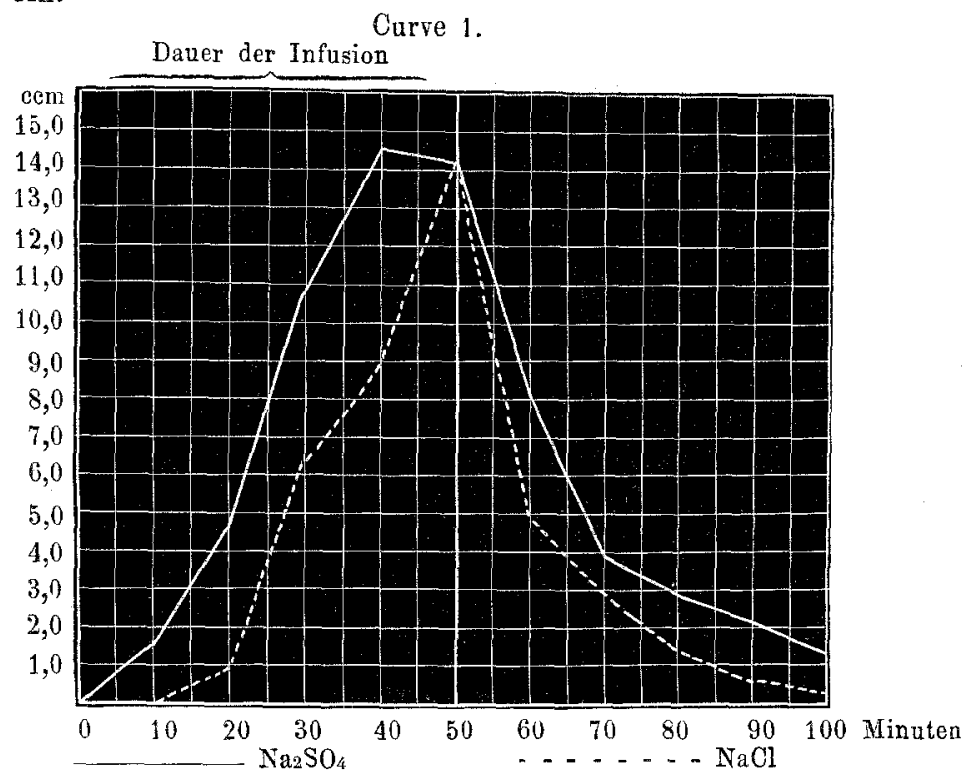

Wie später näher auseinander gesetzt wird, wurden zum Studium der Salzvertheilung im Körper Parallelversuche mit beiden Salzen auch an Hunden durchgeführt. Die nähere Versuchsanordnung wird an der betreffenden Stelle geschildert werden; sie war im Princip die gleiche, nur dass die Einlaufsgeschwindigkeit $0,2 \mathrm{~cm}$ pro Minute and Kilogramm betrug. Hier soll nur angeführt werden, wie sich bei den Hunden die Diurese, die Ausscheidung von Wasser und Salz gestaltet. Es ergaben sich im Ganzen die gleichen Resultate, wie am Kaninchen. Die folgende Tabelle II giebt die näheren Daten.

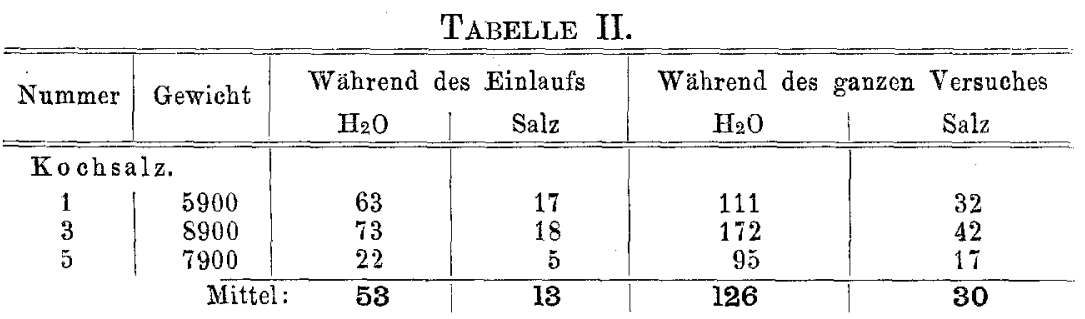


Vergleich der diuretischen Wirksamkeit isotonischer Salzlösungen.

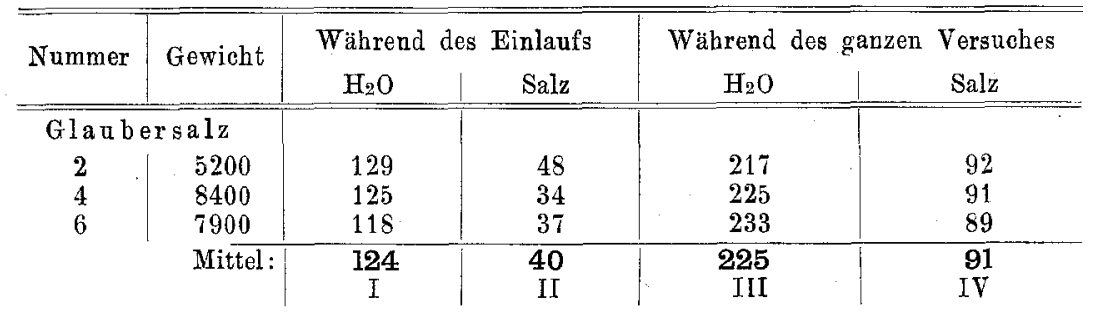

Das Ergebniss zeigt fast noch schlagender, dass das Glaubersalz viel stärker diuretisch wirkt, als das Kochsalz. Bis zum Schluss des Einlaufs (50 Minuten) sind in den Kochsalzversuchen 22 bis 73 Proc. des eingeführten Wassers (Mittel 53 Proc.) und 5-18 Proc. des Salzes (Mittel 13 Proc.) ausgeschieden. In den Glaubersalzversuchen haben 118-129 Proc. des Wassers (Mittel 124 Proc.) und 34-48 Proc. des Salzes (Mittel 40 Proc.) den Körper verlassen. Bei $\mathrm{NaCl-Infusion} \mathrm{blieb} \mathrm{also} \mathrm{die} \mathrm{Ausfubr} \mathrm{hinter} \mathrm{der} \mathrm{Einfuhr} \mathrm{zuriuck,} \mathrm{nach}$ Glaubersalz trat das umgekehrte Verhalten ein. Während des ganzen Versuchs, also in 4 Stunden wurden nach NaCl-Infusion 95-172 Proc. des Wassers (Mittel 126 Proc.) und 17-42 Proc. des Salzes (Mittel 30 Proc.) ausgefibrt. Nach Glaubersalzinfusion 217-233 Proc. des Wassers (Mittel 225 Proc.) und 89-92 Proc. des Salzes (Mittel 91 Proc.). Also auch beim Hund ist die Glaubersalzdiurese fast doppelt so stark, wie die NaCl-Diurese. Auch die Ausfuhr des Salzes ist bei $\mathrm{Na}_{2} \mathrm{SO}_{4}$ viel grösser als bei $\mathrm{NaCl}$.

Der Vergleich von Hund und Kaninchen ergiebt, dass die Wasserausseheidung ungefähr die gleiche ist: im Mittel 131 Proc. beim Kaninchen, 126 Proc. beim Hund nach Kochsalz; und 226 Proc. beim Kaninchen, 225 Proc. beim Hund nach Glaubersalz. Auch rom eingefuhrten Kochsalz scheiden beide Thiere während der Diurese ungefähr das Gleiche (ca. 1/3 der eingeführten Salzmenge) aus: Kaninchen 35 Proc., Hunde 30 Proc. Unterschiede zeigen sich nur im Verhalten gegen Glaubersalz. Am Schluss der Diurese haben die Kaninchen erst 61 Proc. (46-71 Proc.) des Salzes ausgeharnt, Hunde dagegen schon 91 Proc. (89-92 Proc.). - Nach Schluss der Diurese sind bei beiden Thieren also noch ca. ${ }^{2 / 3}$ des Kochsalzes im Körper. Vom Glaubersalz ist beim Kaninchen über 1/3 noch der Ausscheidung entgangen, bei Hunden ist nur der kleine Betrag von $1 / 1_{0}$ des zugeführten Salzes im Körper zurtickgeblieben. Das Glaubersalz scheint also beim Hunde noch harnfähiger qu sein als beim Kaninchen.

Die so deutliche Beziehung, die beim Kaninchen zwischen ausgeschiedener Salz- und Wassermenge sich ergab, ist beim Hunde 
nicht so ausgesprochen. Der Glaubersalzharn ist relativ salzreicher, als der Kochsalzharn. Vielleicht ist die Zahl der Versuche am Hund eine $\mathbf{z u}$ geringe, vielleicht stand bei den Thieren, welche 4 Tage gehungert hatten, eine so grosse Wassermenge (ca. 382 Proc. des Einlaufwassers) nicht zur Verfügung, da wir wissen, wie empfindlich Hunde gegen Wasserentziehung sind.

Die mittlere Salzconcentration des Harnes betrug nach $\mathrm{NaCl}$ : 1,17 Proc., nach $\mathrm{Na}_{2} \mathrm{SO}_{4}: 3,00$ Proc. Die grössten beobachteten Concentrationen betrugen 1,63 Proc. $\mathrm{NaCl}$ und 3,87 Proc. $\mathrm{Na}_{2} \mathrm{SO}_{4}$. Eiweiss fehlte in allen Fällen, Zucker wurde nacb Glaubersalz stets, nach Kochsalz nur einmal beobachtet.

Die bisher beschriebenen Versuche waren in der Weise angestellt, dass Thieren, welche auf gleiche Weise vorbereitet waren, gleiche Mengen Salzlösnng mit gleicher Geschwindigkeit infundirt wurden. Hierbei erhielt ein und dasselbe Thier immer nur eine der beiden Salzlösungen. Die genügend grosse Zahl von Einzelversuchen sichert das Resultat. Immer schien es wünschenswerth und zugleich demonstrabler zu sein, die Verschiedenheit beider Salze an ein und demselben Thier zeigen zu können. Schon Heidenhain (a. a. O.) bat die verschieden stark lymph- und harntreibende Wirkung gleichconcentrirter (nicht isotonischer) Lösungen von $\mathrm{NaCl}$ und $\mathrm{NaI}$ an einem Hunde nachgewiesen, indem er beide nacheinander, jedes mit einer einmaligen kurzen Injection, einführte. Teh bediente mich im Wesentlichen einer Versuchsanordnung, die Herr Dr. E. Rost am k. Gesundheitsamt in Berlin zur systematischen Untersuchung der Salze auf ihren diuretischen Effect angewandt hat und über die er demnächst an anderer Stelle ausführlich berichten wird. Kaninchen welche nicht gehungert hatten, dienten zum Versuche, da hier die Nothwendigkeit, verschiedene Thiere auf gleichen Wasserstand zu bringen, fortfiel. In die Vena jugularis war eine Canüle eingebunden, welche durch ein kurzes T-Robr und kurze Schlanchverbindungen mit 2 Buretten in Verbindung stand. Die eine enthielt NaCl-Lösung, die andere $\mathrm{Na}_{2} \mathrm{SO}_{4}$. Zuerst lief die eine der beiden Salzlösungen mit constanter Gesehwindigkeit ein. Sobald die Diurese eine gleichmässige geworden $\mathrm{war}_{9}$, wurde die erste Burette geschlossen und die zweite geöffnet. War hiernach die Diurese deutlich geändert, so wurde wieder die erste Salzlösung infundirt, worauf die Harnfluth sich wieder dem alten Werth näherte. Diese Versuche sind insofern complicirter, als bei der Zufuhr des zweiten Salzes noch ein beträchtlicher Theil des ersten im Blute kreist, also 
die Wirkung nicht die reine Wirkung eines einzelnen Salzes ist. Doch sind die Ausschläge so deutlich, dass an ihrer Beweiskraft nicht gezweifelt werden kann. Das zeigen die folgenden Diagramme, welche die Harnmengen in je 5 Minuten angeben. Die Glaubersalzperioden sind sehraffirt gezeichnet:

Curve 2.

Kaninchen, $1950 \mathrm{~g}$. Einlaufsgeschw. 0,26 pro Min. und Kg. Zuerst $\mathrm{Na}_{2} \mathrm{SO}_{4}$, dann $\mathrm{NaCl}$, dann $\mathrm{Na}_{2} \mathrm{SO}_{4}$.

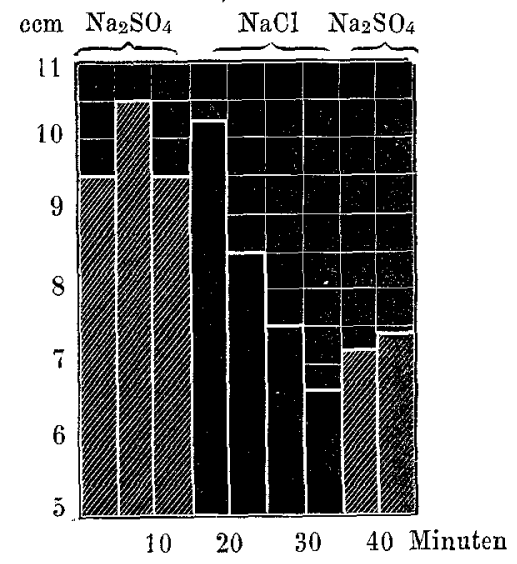

Curve 3 .

Kaninchen, 1800 g. Einlaufsgeschw. 0,28 pro Min. und $\mathrm{Kg}$. Znerst $\mathrm{NaCl}$, dann $\mathrm{Na}_{2} \mathrm{SO}_{4}$, dann $\mathrm{NaCl}$.

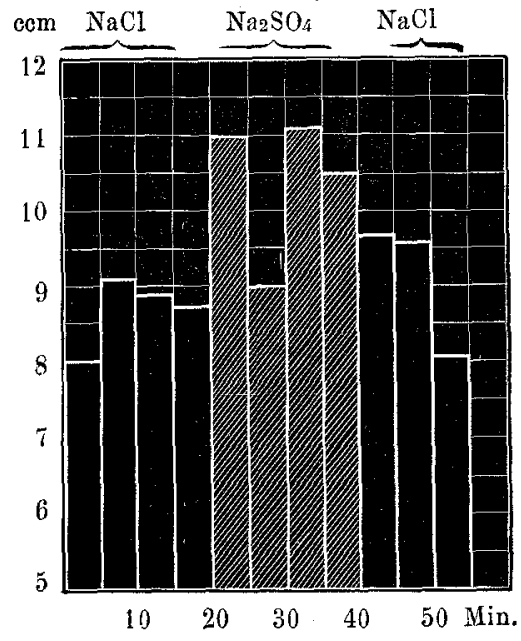

Man sieht, dass in dem ersten Versuch (Fig. 2) während einer auf Glaubersalz folgenden NaCl-Zufuhr die Diurese (nachdem in den ersten 5 Minuten der Zwischenperiode, in weloher erst alles $\mathrm{Na}_{2} \mathrm{SO}_{4}$ aus dem T-Rohr und den Schlauchstücken verdrängt werden muss, die Harnmenge noch hoch bleibt) alsbald absinkt, um nach abermaliger $\mathrm{Na}_{2} \mathrm{SO}_{4}$-Infusion wieder zu steigen. - Im zweiten Versuche erfolgt bei der auf einer constanten NaCl-Diurese folgenden Zufuhr der isotonischen $\mathrm{Na}_{2} \mathrm{SO}_{4}$-Lösung sofort ein Ansteigen der Harnfluth, die auf abermalige Kochsalzzufuhr schnell wieder absinkt. Es lässt sich also auch am selben Thier der Vergleich beider Salzlösungen durchführen mit dem klaren Ergebniss, dass Glaubersalz stärker diuretisch wirkt als Koebsalz.

Es konnte in diesem ersten Abschnitt gezeigt werden, dass isotonische Lösungen verschiedener Salze ( $\mathrm{NaCl}$ und $\mathrm{Na}_{2} \mathrm{SO}_{4}$ ) unter sonst ganz gleichen Bedingungen bei intravenöser Einfuhr verschiedene diuretische Wirkung haben, dass bei dem stärker wirksamen, 
dem Glaubersalz in der Zeiteinheit nicht nur die Wasserausseheidung, sondern auch die Salzausfuhr eine grössere ist, dass, wenigstens beim Kaninchen, beide Salze im Allgemeinen in nahezu gleichem Proc.Verbältniss zur Wassermenge producirt werden, dass vom Glaubersalz am Schluss der Diurese ein kleinerer Bruchtheil im Körper zurückbleibt als beim Kochsalz, und dass dieser Bruchtheil beim Hunde $\mathrm{kleiner}$ ist als beim Kaninchen.

In der Einleitung war die Frage aufgeworfen, ob die diuretische Wirkung starker Salzlösungen allein zu erklären sei aus der Thatsache, dass diese Lösungen einen Strom von Wasser ans den Geweben ins Blut hervorrufen, also Blutverdunnung, hydrämische Plethora erzengen. Es musste sich daraus die Frage ergeben: Beruht die stärkere Wirkung des Glaubersalzes darauf, dass es stärkere Blutverdünung hervorruft als das Kochsalz? Oder aber, ist die Blutverdünnung in beiden Fällen die gleiche, und trotzdem die diuretische Wirksamkeit versehieden? Die Beantwortung dieser Frage musste zugleich die Entseheidung liefern, ob wirklich die hydrämische Plethora der wichtigste oder einzigste Faktor für die Salzdiurese ist. Ihr ist der folgende Abschnitt gewidmet.

\section{B. Die Blutverdünnung während der Diurese.}

Wie sehon oben erwähnt ist, wurde den Kaninchen und ebenso den Hunden dreimal je ein Tropfen Blut zur Hämoglobinbestimmung: entnommen, und zwar vor Beginn des Einlaufs, unmittelbar nach Schluss desselben und am Schluss des ganzen Versuches, also nach 4 Stunden. Diese Bestimmung diente dazu, die Veränderungen des Wassergehaltes im Blute za berechnen. Um eine etwaige Blutverdünnung im Blute nachweisen zu können, muss man von einem Blutbestandtheil ausgehen, der während des ganzen Versuches die Blutbahn nicht in nachweisbaren Mengen verlässt. Dieses sind, wie schon in der "I. Mittheilung" ausführlich begründet wurde, weder der Gesammttrockengehalt des Blutes, noch sein Eiweissgehalt, sondern allein die Erythrocyten, bezw. der in ihnen enthaltene Blutfarbstoff. Ist nun z. B. der Hämoglobingehalt des Blutes vor dem Einlauf 20 Proc., nach Schluss desselben 10 Proc., so muss, da sich die absolute Menge Blutfarbstoff in der Gefässbahn nicht geändert hat, das Blut um das Doppelte verdünnt worden sein. Die Blutverdinnung beträgt 100:200. Der Kürze wegen sei im Folgenden eine Blntverdünnung, bei der dieselbe Menge Hämoglobin, die früher in 100 Theilen Blut enthalten war, nun zum Beispiel in 116 Teilen 
Vergleich der diuretischen Wirksamkeit isotonischer Salzlösungen. 409

Blut sich befindet, als Blutverdünnung 116 bezeichnet. Es sind dann je 100 Theile Blut auf 116 Theile verdünt worden.

Will man hieraus die absoluten bewegten Wassermengen berechnen, so muss man eine Annahme einführen, die Schätzung der Blatmenge des Thieres vor dem Versuch. Dieselbe wird im Folgenden zu 7 Proc. des Körpergewichts angenommen. (Die Begründung hierfür sowie die ausfübrliche Darlegung der ganzen Berechnungsweise ist bereits eingehend in der "I. Mittheilung" gegeben, so dass hier darauf verwiesen werden kann, a. a. 0. S. 73-81. Beträgt z. B. die ursprüngliche Blutmenge 300 , die Blutverdünnung 125, so sind aus je 100 Blut I nunmehr 125 Blut II geworden, also aus 300 Blut I jetzt 375 Blut II. Die Wasserzunahme des Blutes beträgt demnach $75 \mathrm{ccm}$. Da die Ausscheidungen bekannt sind, so lässt sich anch der Wasseraustausch zwischen Blnt und Gewebe unmittelbar berechnen. Das Resultat dieser Auswerthungen giebt für die Versuchsreibe an Kaninchen die folgende Tabelle III, für die Hundeversuche die Tabelle IV. Der Zeitpunkt der Blutentnahmen vor dem Versuch ist als Moment I, der Schluss des Einlaufs ist als Moment II, der Schluss des ganzen Versuches als Moment III bezeichnet.

TABelle III.

\begin{tabular}{|c|c|c|c|c|c|c|c|c|}
\hline \multirow[b]{2}{*}{$\mathrm{Nr}$. } & \multirow[b]{2}{*}{$\begin{array}{c}\text { Ge- } \\
\text { wicht }\end{array}$} & \multicolumn{3}{|c|}{ Von Moment I-II } & \multicolumn{2}{|c|}{ Von Moment II-III } & \multirow{2}{*}{$\begin{array}{l}\text { Ganzer } \\
\text { Diurese } \\
\text { in Proe. } \\
\text { des } \\
\text { Einlaufs }\end{array}$} & \multirow{2}{*}{$\begin{array}{l}\text { Versuch } \\
\begin{array}{|c}\text { Gesammt- } \\
\text { wasser- } \\
\text { verlust der } \\
\text { Gewebe }\end{array}\end{array}$} \\
\hline & & $\begin{array}{c}\text { Diurese } \\
\text { in Proc. } \\
\text { des } \\
\text { Einlaufs }\end{array}$ & $\begin{array}{c}\text { Blut- } \\
\text { ver- } \\
\text { dünnung }\end{array}$ & $\begin{array}{c}\text { Aus dem } \\
\text { Gewebe } \\
\text { ins Blut } \\
\text { com }\end{array}$ & $\begin{array}{c}\text { Blut- } \\
\text { ver- } \\
\text { dtinnung }\end{array}$ & $\begin{array}{c}\text { Aus dem } \\
\text { Gewebe } \\
\text { ins Blut } \\
\text { com }\end{array}$ & & \\
\hline \multicolumn{3}{|c|}{ Kochsalz: } & & & & & & \\
\hline 5 & 1640 & 67 & 111 & $-0,9$ & 101 & $+0,8$ & 97 & $-0,1$ \\
\hline 6 & 1735 & 97 & 129 & $+33,5$ & 104 & $+4,3$ & 176 & $+37,8$ \\
\hline 7 & 1600 & 38 & 120 & $-5,6$ & 100 & $-14,1$ & 50 & $-19,7$ \\
\hline 8 & 1160 & 57 & 122 & $+5,0$ & 102 & $-5,3$ & 93 & $-0,3$ \\
\hline 9 & 1850 & 122 & 110 & $+11,3$ & 101 & $+20,3$ & 167 & $+31,6$ \\
\hline 10 & 1840 & 125 & 123 & $+41,1$ & 105 & $+4,5$ & 184 & $+45,6$ \\
\hline 19 & 1400 & 93 & 110 & $+7,0$ & 109 & $+19,4$ & 149 & $+26,4$ \\
\hline & Mittel: & 85 & 118 & & 103 & & 131 & $+17,3$ \\
\hline \multicolumn{3}{|c|}{ Glaubersalz: } & . & & & & & \\
\hline 11 & 1470 & 73 & 112 & $+3,0$ & 85 & $+6,7$ & 169 & $+9,7$ \\
\hline 12 & 1620 & 162 & 109 & $+35,0$ & 96 & $+5,4$ & 210 & $+40,4$ \\
\hline 14 & 2150 & 104 & 115 & $+30,5$ & 104 & $+31,4$ & 210 & $+64,9$ \\
\hline 15 & 1920 & 182 & 105 & $+46,1$ & 91 & $+26,7$ & 277 & $+72,8$ \\
\hline 16 & 1300 & 151 & 127 & $+40,7$ & 100 & $+2,7$ & 233 & $+43,4$ \\
\hline 18 & 1450 & 164 & 128 & $+51,9$ & 101 & $+4,6$ & 254 & $+56,5$ \\
\hline \multirow{2}{*}{\multicolumn{2}{|c|}{ Mittel: }} & 139 & 116 & & 96 & & 226 & +48 \\
\hline & & I & II & III & IV & $\mathrm{V}$ & VI & VII \\
\hline
\end{tabular}


TABelle IV.

\begin{tabular}{|c|c|c|c|c|c|c|c|c|}
\hline \multirow[b]{2}{*}{$\mathrm{Nr}$} & \multirow[b]{2}{*}{$\begin{array}{l}\text { Gre- } \\
\text { wicht }\end{array}$} & \multicolumn{3}{|c|}{ Von Moment I-II } & \multicolumn{2}{|c|}{ Yon Moment II-III } & \multirow{2}{*}{$\begin{array}{l}\text { Ganzer } \\
\text { Diurese } \\
\text { in Proc. } \\
\text { des } \\
\text { Einlaufs }\end{array}$} & \multirow{2}{*}{$\begin{array}{l}\text { Versuch } \\
\text { Gesammt- } \\
\text { wasserver- } \\
\text { lust der } \\
\text { Gewebe }\end{array}$} \\
\hline & & $\begin{array}{c}\text { Diurese } \\
\text { in Proe } \\
\text { des } \\
\text { Einlanfs }\end{array}$ & $\begin{array}{c}\text { Blut- } \\
\text { ver- } \\
\text { dûnnung }\end{array}$ & $\begin{array}{c}\text { Aus den } \\
\text { Geweben } \\
\text { ins Blut } \\
\text { ccm }\end{array}$ & $\begin{array}{l}\text { Blut- } \\
\text { ver- } \\
\text { dünnung }\end{array}$ & $\begin{array}{l}\text { Aus den } \\
\text { Geweben } \\
\text { ins Blut } \\
\text { cen }\end{array}$ & & \\
\hline \multicolumn{3}{|c|}{ Kochsalz: } & & & & & & \\
\hline 1 & 5900 & 63 & 105 & -4 & 107 & +34 & 111 & +30 \\
\hline 3 & 8900 & 73 & 128 & +110 & 100 & -37 & 172 & +73 \\
\hline 5 & 7900 & 22 & 121 & +47 & 110 & +4 & 75 & +51 \\
\hline \multicolumn{2}{|c|}{ Mittel: } & 53 & 116 & & 106 & & 126 & +51 \\
\hline \multicolumn{3}{|c|}{ Glaubersalz: } & & & & & & \\
\hline 2 & 5200 & 129 & 154 & +187 & 93 & -129 & 217 & +57 \\
\hline 4 & 8400 & 125 & 94 & -13 & 85 & +76 & 225 & +63 \\
\hline 6 & 7900 & 118 & 115 & +89 & 91 & -21 & 233 & +68 \\
\hline \multicolumn{2}{|c|}{ Mittel: } & 124 & 121 & & 90 & & 225 & +63 \\
\hline & & I & II & III & IV & $\mathrm{V}$ & $\mathrm{VI}$ & VII \\
\hline
\end{tabular}

Betrachten wir zunäelst das Verhalten der Blutverdünnung beim Kaninchen (Tab. III. Col. II), so sehen wir, dass auf der Höhe der Diurese, also im Moment II, in allen Fällen Blutverdünnung vorhanden ist. Diese schwankt beim Kochsalz von $110-129$ und beträgt im Mittel 118, beim Glaubersalz von 105-128 und beträgt im Mittel 116. Der Mittelwerth für die Glaubersalzversuche ist also sogar etwas niedriger wie für die Kochsalzversuche. Beim Hund (Tab. IV. Col. II) beträgt die Blutverdünnnng im Mittel der Kochsalzversuche 116, der Glaubersalzversuche 121. Hier ist der letztere Werth um ein kleines höher. Zieht man aus den Kaninchen- und Hundeversuchen die gemeinsame Mittelzahl, so ergiebt sich fur $\mathrm{NaCl}$ eine Blutverdünnung auf der Höhe der Diurese von 117,4, während derselbe Werth für $\mathrm{Na}_{2} \mathrm{SO}_{4} 117,7$ beträgt.

Das heisst mit anderen Worten: Nach Einlauf isotonischer Kochsalz- und Glaubersalzlösungen tritt durchsehnittlich die gleiche Blutverdün $n$ ung auf. Die verschieden grosse Ditrese kann also nicht von einer Verschiedenheit der Blutverdünnung abgeleitet werden. Wir sind jetzt im Stande, die in der Einleitung aufgeworfene Frage zu beantworten: Verschiedene Salzlösungen, welche gleiche Blutverdünung lervorrufen, können trotzdem verschieden starke Diurese bedingen. Es kann also die Blutverdünnug niebt der einzige Faktor 
sein, welcher die Diurese nach Infusion starker Salzlösungen hervorruft. Vielmehr müssen noch andere Momente mitspielen. ${ }^{1}$ )

Ebenso wie in den in der ersten Mittheilung beschriebenen Versuchen sehen wir nun nach Sohluss der Infusion die Blutverdünnung mit absinkender Diurese zurïckgehen. Die Werthe, die am Schluss der Versuche gefunden wurden, giebt Col. IV. in Tab. III und IV. In den Kochsalzversuchen beträgt sie nach 4 Stunden noch 100 bis 109, im Mittel 103 bei Kaninchen, und 106 beim Hund. Es ist also nach dem Absinken der Diurese noch ein geringer Grad von Blutverdünnung vorhanden. Anders in den Glaubersalzversuchen. Beim Kaninchen sehen wir, dass in der Hälfte der Fälle, beim Hunde in allen Fällen am Schluss der Diurese das Blut nicht nur seine alte Concentration wieder erlangt hat, sondern sogar eingedickt ist. Die Mittelwerthe sind für Kaninchen 96, für Hunde 90. Hier hat also, wie das auch Starling für einige Fälle beschreibt, die Diurese die Blutverdünnung überdanert und hat zu einer Verarmung des Blutes an Wasser geführt. Dass bei Glaubersalzinfusion dieses ev. schon sehr frühe eintreten kann, zeigt der Versuch am Iund Nr. 4 (Tab. IV Col. II), bei dem eine derartige Bluteindickung bis auf 94 bereits auf der Höhe der Diurese nachweisbar war, welche sich dann bis zum Sehluss des Experiments (Col. IV) bis auf 85 steigerte.

Diese Ergebnisse bekräftigen das oben erhaltene Resultat, dass die Blutverdünnung nicht das einzige Movens für die Salzdiurese sei, in erfreulicher Weise. Wir sehen, dass ein Etwas die Niere treiben muss, fort zu secerniren, wenn auch keine Blutverdünnung mehr vorhanden ist, und dass dieses Etwas nach Glaubersalzzufuhr wirksamer ist, als nach Kochsalzinfusion.

Auf welche Weise kommt nun die soeben skizzirte Blutverdünnung zu Stande? Hierüber geben die Zahlen über die Wasserbewegung zwischen Blut und Gewebe Aufschluss (Tab. III und IV, Col. III, V, VII). Das Blut hat in diesen Versuchen zwei Quellen für. seinen vermehrten Wassergehalt: Erstens die Infusionsflüssigkeit und zweitens das Gewebswassei. Es kann anf zweierlei Weise Wasser abgeben, erstens durch die Nieren und zweitens wieder zuritck an

1) Dass nach Einfuhr isotonischer Salzlösungen gleiche Blutverdünnung auftritt, ist zugleich der beste Beweis dafür, dass die gewählte Art, physiologisch gleichwerthige Lösungen berzustellen, die richtige war; dass man recht daran gethan hat, isotonische Lösungen zum Vergleich zu nehmen und nicht etwa Lösungen von gleichem Gehalt an $\mathrm{Na}$, oder event. an Säureadikalen allein. 
die Gewebe. In der I. Mittheilung ist ausführlich gezeigt worden, dass nach Einlauf verdünnter Salzlösungen (die dem Blut gegenuiber iso- oder bypotoniseh waren) ein Theil des Einlaufswassers die Blutverdünnung herbeiführt, ein anderer grösserer 'Theil schnell in den Geweben deponirt wird, von wo er dann später zum Theil in das Blut zurückfliesst, um durch die Niere ausgeschieden zu werden. Nach Einfuhr concentrirter Kochsalzlösung (35 Proc.) bezieht dagegen das Blut sein Wasser aus den Geweben und wird dadurch verdünnter. In den in der vorliegenden Arbeit geschilderten Versuchen kamen Salzlösungen in Verwendung, deren Concentration zwischen jenen beiden Extremen in der Mitte steht. Wir sehen demnach ein verschiedenes Verhalten. In der grösseren Mehrzahl der Experimente genügt auf der Höhe der Diurese (Col. III) das Infusionswasser nicht, um sowohl die Diurese als auch die Blutverdünnung zu bestreiten, es tritt demnach Wasser aus dem Gewebe in die Blutbahn (positives Vorzeichen). Bei 4 Thicren (Kaninchen 5 und 7 , Hund 1 und 4) ist dagegen noch Wasser aus der Blutbahn in die Gewebe gegangen. Nach Schluss des Einlaufs wird dem Körper kein Wasser mehr von aussen zugeführt. Er verliert dagegen continuirlich Wasser durch die Niere. Dieses Wasser wird zunächst aus dem Blute entnommen, und wir sehen demnach im zweiten Theil des Versuches die Blutverdünnung zurickgehen. Ausserdem strömt aber in der Mebrzahl der Versuche Wasser aus den Geweben ins Blut nach (Col. V. positives Vorzeichen). In einer Minderzahl dagegen entledigt sich das Blut seines Wasserubberschusses nicht nur durch die Nieren, sondern auch in die Gewebe (negatives Vorzeichen). Es sind dies zwei Kaninchen (Nr. 7 und 8), bei denen die Diurese (Col. VI) eine sehr schwache, unter 100 Proc. des Einlaufs betragende ist; und ausserdem 3 Hunde ( $N r .2,3$ und 6), bei denen im ersten Theil des Versuches ein iiberaus reichlicher Wasserstrom ans dem Gewebe ins Blut zu dem Einlaufswasser hinzu statt hatte, dass dadurch die starke Diurese voll gedeckt werden konnte. Der Ueberschuss ging dann in die Gewebe zurück.

Col. VII giebt den Endeffect des ganzen Vorgangs, den Gesammtwasserverlust der Gewebe während des ganzen Versuches an. Nur in den 3 Kaninchenversuchen mit Kochsalz (Nr. 5, 7, 8), in denen das Harnquantum hinter der Infusionsmenge zurückblieb, haben die Gewebe kein Wasser verloren, in allen anderen Versuchen aber hat eine Austrocknung stattgefunden. Dieser Wasserverlust der Gewebe ist bei Glaubersalz grösser als bei Kochsalz, und zwar wird bei Kaninchen durch Glaubersalz eine viel stärkere 
Austrocknung der Gewebe herbeigeführt als durch Kochsalz, während bei Hunden die Unterschiede nicht so beträchtlich sind, weil hier das Blut einen grösseren Theil des Wasserverlustes durch Glaubersalz bestreitet (Col. IV).

Es hat sich in diesem Abschnitt ergeben, dass unter den eingehaltenen Versuchsbedingungen isotonische Lösungen von Kochsalz und Glaubersalz gleiche Blutverdinnung herrorrufen; dass demnach die verschiedene diuretische Wirkung beider Substanzen nicht darauf beruhen kann, dass der Niere in einem. Falle ein wasserreicheres Blut vorgesetzt wird als im anderen.

Das Nächstliegende war jetzt, zu untersuchen, ob, wenn auch die Wasservertheilung keine Unterschiede erkennen liess, die Vertheilung der beiden Salze im Körper nicht eine verschiedene wäre, etwa so, dass von dem eingefübrten Glanbersalz ein grösserer Theil im Blute kreiste als vom Kochsalz; und dass dadurch die versehieden starke Diurese erklärt werden könnte.

Der Beantwortung dieser Frage dienten die im nächsten Absehnitt beschriebenen Versuche.

\section{Die Salzvertheilung während der Diurese.}

Die Experimente wurden an 6 Hunden angestellt, und es ist im Vorhergehenden schon mehrfach von den Ergebnissen, die an diesen Thieren betr. Diurese und Blutverdünnung gewonnen wurden, die Rede gewesen. Hier soll demnach nur noch auf die Salzvertheilung eingegangen werden.

Die Hunde hungerten und dursteten je 4 Tage vor dem Versuch, um sie auf annähernd gleichen Wasserstand zu bringen. In tiefer Morphinnarkose wurde ihnen $45 \mathrm{ccm}$ Blut aus der Carotis für die Analysen entnommen und 10 Minuten lang die Normalharnsecretion aus Ureterenkanülen bestimmt. Darauf lief in die Jugularis eine der beiden Salzlösungen mit einer Einlaufsgeschwindigkeit ron 0,2 pro Min. und $\mathrm{kg}$ (statt 0,5 beim Kaninchen, da Hunde gegen derartige Eingriffe empfindlicher zu sein scheinen 1) 50 Min. lang ein, also im Ganzen $10 \mathrm{cem}$ pro kg. Am Schluss des Einlaufs (Moment II) wurde ein zweiter und nach weiteren 3 Stunden (Moment III) ein dritter Aderlass von derselben Grösse gemacht. Unmittelbar vor jedem Aderlass wurde ein Tropfen Blut für die $\mathrm{Hb}$.

1) Dastre und Loye, Arch. d. Phys. norm. et pathol. 1888. S. 73 und 1889. S. 253. 
bestimmung entnommen. Während des ganzen Versuches wurde der Urin aufgefangen, für je $10 \mathrm{Min}$. gemessen und in zwei Portionen vereinigt, welehe die vor und nach Schluss der Infusion producirten Harnmengen enthielten. Im Harn wurde der Gehalt an Kochsalz und bei den Glaubersalzversuchen auch an $\mathrm{Na}_{2} \mathrm{SO}_{4}$ bestimmt. Das Blut wurde centrifugirt und im Serum der Gehalt an Kochsalz resp. Glaubersalz sowie die Gefrierpunkterniedrigung ermittelt. Die Begründung der Versuchsanordnung, die Controllversuche über die Wirkung der Aderlässe, sowie die ganze Berechnung der relativen und absoluten Salz- und Wassermengen, die zu einer Salz und Wasserbilanz des Körpers während des Versuches führen, sind in der ersten Mittheilung so ausfulhrlich dargestellt, dass hier darauf verwiesen werden kann (S. 71-82). Im Anbang sind die Analysenzahlen dieser Versucbe tabellarisch aufgeführt. Die Berechnung dürfte darnach leicht durchzuführen sein.

Zunächst zeigt sich, dass die stärkere Diurese, die nach Glaubersalz eintritt, jedenfalls nicht davon abhängig sein kann, dass der Gesammtsalzgehalt des Blutes, resp. der Gehalt an osmotisch wirksamen Molekülen und Ionen höher ist als in den Kochsalzversuchen, vielmehr ist das Gegentheil der Fall. Die folgende Tabelle V zeigt, um wieviel Grad C. der Gefrierpunkt des Blutes am Schluss des Einlaufs (II) und am Ende des Versuches (III) gegen die Norm gesunken ist.

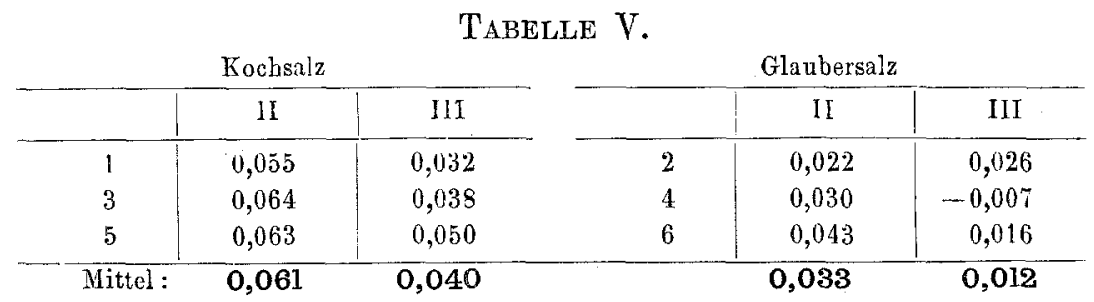

Es zeigt sich also, dass sowohl auf der Höhe als nach Schluss der Diurese das wasseranziehende Vermögen des Blutes durch die Kochsalzinfusion in stärkerem Maassstabe heraufgesetzt wird als durch Glaubersalzeinlauf. Es findet demnach hier die stärkere (Glaubersalz)-diurese bei niedrigerem osmotischen Druck des Blutes statt. Man sieht, dass das wasseranziehende Vermögen des Blutes ohne Einfluss auf die Grösse der Harnfluth ist, wie das schon in der ersten Mittheilung hervorgehoben wurde (S. 99-101).

Wie sich das infundirte Salz im Körper vertheilt und wie sich die quantitativen Verhältnisse dabei gestalten, darüber giebt die nachstehende Tabelle Aufschluss. 
TABElle VI.

Es sind im Procent des infundirten Salzes

\begin{tabular}{|c|c|c|c|c|c|c|c|c|}
\hline \multirow{2}{*}{$\mathbf{N r}$. } & \multirow{2}{*}{$\begin{array}{c}\text { Ge- } \\
\text { wicht }\end{array}$} & \multicolumn{3}{|c|}{ Bis zu Moment II } & \multicolumn{2}{|c|}{ Von Moment II-III } & \multicolumn{2}{|c|}{ Im Moment III } \\
\hline & & $\begin{array}{l}\text { im Harn } \\
\text { ausge- } \\
\text { schieden }\end{array}$ & im Blut & $\begin{array}{l}\text { in die } \\
\text { Gewebe } \\
\text { getreten }\end{array}$ & $\begin{array}{l}\text { im Harn } \\
\text { ausge- } \\
\text { schieden }\end{array}$ & $\mid \begin{array}{c}\text { noch in } \\
\text { dieGewebe } \\
\text { getreten }\end{array}$ & im Blut & $\begin{array}{l}\text { im } \\
\text { Körper }\end{array}$ \\
\hline \multicolumn{9}{|c|}{ Kochsalz: } \\
\hline 1 & 5900 & 17 & 10 & 73 & 15 & -16 & 11 & 68 \\
\hline 3 & 8900 & 18 & $3 \pi$ & 45 & 24 & +3 & 10 & 58 \\
\hline 5 & 7900 & 5 & 37 & 58 & 12 & +4 & 21 & 83 \\
\hline \multicolumn{2}{|c|}{ Mittel: } & 13 & 29 & 58 & 17 & -3 & 14 & 70 \\
\hline \multicolumn{9}{|c|}{ Glaubersalz: } \\
\hline 2 & 5200 & 48 & 28 & 24 & 44 & -16 & 0 & 8 \\
\hline 4 & 8400 & 34 & 11 & 55 & 57 & -47 & 1 & 9 \\
\hline 6 & 7900 & $3 i$ & 25 & 38 & 52 & -27 & 0 & 11 \\
\hline \multirow{2}{*}{\multicolumn{2}{|c|}{ Mittel: }} & 40 & 21 & 39 & 51 & -30 & 0 & 9 \\
\hline & & I & II & III & IV & $V$ & VI & VII \\
\hline
\end{tabular}

Aus diesen Zahlen erhellt zunächst, dass die stärkere Diurese an Glaubersalzinfusion nicht darauf beruhen kann, dass im Blut von dem Glaubersalz ein grösserer Theil kreist als vom Kochsalz. In Col. II ist angegeben, wieviel von dem im Ganzen eingefiuhrten Salze auf der Höhe der Diurese in der Blutbahn sich befindet. In den Kochsalaversuchen sind dies 10-37 Proc., im Mittel 29 Proc., in den Glaubersalzversuchen 11-28 Proc., im Mittel 21 Proc. Es ist also von dem eingeführten $\mathrm{NaCl}$ ein grösserer Bruchtheil im Blut als vom Glaubersalz. Und trotzdem tritt nach $\mathrm{Na}_{2} \mathrm{SO}_{4}$ die höhere Diurese ein. Col. III zeigt, dass ein grosser Theil des eingeführten Salzes die Blutbahn verlässt und in die Gewebe tritt. Da bis Schluss des Einlaufs durch die Niere bereits mehr Glaubersalz aus dem Körper herausbefördert ist als Kochsalz (Col. I), so ist der Theil, der in die Gewebe geht, bei Kochsalz grösser (im Mitfel 58 Proc.) als bei Glaubersalz (im Mittel 39 Proe. des eingeführten). Die Salzlösungen bewirken also, wie schon Klikowicz und Münzer betonten, dass Wasser in die Blutbahn tritt und dafür Salz in die Gewebe geht. Nach Schluss des Einlaufs ist das Verhalten ein verschiedenes (Col. V). In zwei Kochsalgversuchen (Nr. 3 und 5) ist auch weiterhin ein Uebertritt von Kochsalz in die Gewebe zu constatiren. In Kochsalzversuch 2 dagegen und in allen Glaubersalzversuchen ist das Gegentheil der Fall. Hier ist die Niere das einzige Organ, welches noch Salz aus der Blutbahn herausbefördert, der Salzstrom zwischen Blut und Gewebe hat sich umgekehrt, es 
hat sich jetzt Salz aus dem Gewebe ins Blut zurïckbewegt, um schliesslich durch die Nieren ausgeschieden zu werden.

Das Endergebniss ist bei den beiden Salzen ein verschiedenes. Am Schluss der Diurese sind in den Kochsalzversuchen im Mittel noch 70 Proc. des eingeführten Salzes im Körper (Col. VII). Davon sind 14 Proc. in der Blutbahn (Col. VI), gleichzeitig mit einer noch bestehenden Blutverdünnung von 103, und 56 Proc. in den Geweben. Es ist also über die Hälfte des überhaupt eingeführten Salzes noch in den Geweben.

Beim Glaubersalz dagegen ist der Endeffect ein anderer. Es bleibt am Schluss des Versuches weniger als 1/10 der Gesammtsalzmenge im Körper, und zwar fast ausschliesslich in den Geweben, während das Blut beinahe frei davon geworden ist (Col. VI und VII).

Während also bis zur endgültigen Ausseheidung nach Kochsalzeinlauf die Gewebe für längere Zeit Salz in sich deponiren müssen, wird nach Glaubersalzzufuhr nur fur wenige Stunden das Salz in die Gewebe geworfen. Dann wandert es wieder zurück ins Blut und verlässt durch die Niere den Körper.

Es zeigt sich also, dass in den beschriebenen Parallelversuchen die Niere in gleicher Zeit viel grössere Mengen Glaubersalz befördert als Kochsalz. Sie verhält sich mithin umgekehrt wie die Darmwand, welche bekanntlich $\mathrm{Na}_{2} \mathrm{SO}_{4}$ viel langsamer und weniger resorbirt als NaCl. - Die Wände der Capillaren zeigen gegenüber dem Durchtritt beider Salze keine wesentlichen Verschiedenheiten. Beide gehen sehr leicht in die Gewebe, die Blutverdünnung, welche beide Salze in isotoniseher Lösung hervorrufen, ist die gleiche und die Vertheilung von Blut und Gewebe lässt auch keine wesentlichen Abweichungen erkennen. Die folgende Tabelle zeigt, ein wie grosser Procentsatz des überhaupt noch im Körper befindlichen Salzes sich am Schluss des Einlaufs in den Geweben befindet.

Tabelle VII.

\begin{tabular}{|c|c|}
\hline \multicolumn{2}{|c|}{ Koohsalz } \\
\hline Nummer & im Gewebe \\
\hline $\mathfrak{1}$ & 88 \\
\hline 3 & 55 \\
\hline 5 & 61 \\
\hline Mittel: & 68 \\
\hline
\end{tabular}

\begin{tabular}{c|c}
\multicolumn{2}{c}{ Glaubersalz: } \\
\hline Nummer & im Gewebe \\
\hline 2 & 46 \\
4 & 83 \\
6 & 60 \\
\hline Mittel : & $\mathbf{6 3}$
\end{tabular}

Man sieht, dass im Mittel sich 68 Proc. des Kochsalzes gegen 63 Proe. des Glaubersalzes in die Gewebe begeben haben, also ungefäbr die gleichen relativen Mengen. Es müssen also die Capillarwände dem Durchtritt der beiảen Salze nicht wesentlich versehiedene 
Widerstände entgegensetzen. Das soeben geschilderte lässt sich kurz vielleicht so ausdrücken: Während der Darm für Glaubersalz viel sehwerer durchgängig ist, als für Kochsalz, ist die Niere im Gegentheil leichter durehgängig für Glaubersalz, und die Gefässwand verhält sich beiden Salzen gegenüber etwa gleich.

Dass der Darm auch in entgegengesetzter Richtung für Glaubersalz schwer durchgängig ist, zeigte die Bestimmung des $\mathrm{Na}_{2} \mathrm{SO}_{4}$ im Magen- und Darminhalt nach Schluss des Versuches, welche (die Thiere hatten 4 Tage gehungert) vollständig negativ ausfiel. Doch wird auch $\mathrm{NaCl}$ nicht in wesentlichen Mengen in den Darm ausgeschieden, wie Münzer gezeigt hat. Eine Bestimmung, die in unserem Versuch 5 ausgeführt wurde, ergab im Magendarminhalt 0,533 Proe. $\mathrm{NaCl}=0,33 \mathrm{~g}$, von dem wohl nur ein Theil der infundirten Salzmenge entstammte, da immer Kochsalz im Darm zu finden sein dürfte.

Interessant gestaltete sich auch in den besprochenen Versuchen der Vergleich zwischen dem Salzgehalt des Blutes und Harns. $\mathrm{Hamburger}{ }^{1}$ ) hat nachgewiesen, dass nach Infusion von $\mathrm{Na}_{2} \mathrm{SO}_{4}$ Lösung das wasseranziehende Vermögen des Blutes nicht so stark steigt, wie der Zunahme an Glaubersalz entspricht, dass also noch andere Bestandtheile des Plasmas zusammen mit einem Theil des $\mathrm{Na}_{2} \mathrm{SO}_{4}$ die Blutbahn verlassen müssen, und er fand, dass in der. That der proc. NaCl-Gehalt des Serums gesunken war. - So sehen wir auch z. B. in unserem Versuch 4, dass durch den Einlauf der $\mathrm{Na}_{2} \mathrm{SO}_{4}$-Gehalt des Blutes von 0,034 Proc. auf 0,272 Proc. stieg, der $\mathrm{NaCl}$-Gehalt aber von 0,632 Proc. auf 0,600 Proc. sank. Wie die quantitative Berechnung ergiebt, beruht dieser Kochsalzverlust nicht auf der Harnausscheidung und auch nicht darauf, dass Wasser aus dem Gewebe ins Blut tritt und den proc. Werth herabdrückt, sondern es haben in der That während des ganzen Versuches $0,4 \mathrm{~g}$ Kochsalz die Blutbahn verlassen und sind ins Gewebe getreten.

Wie stellt sich nun unter diesen Umständen, bei gesunkenem Proc. Kochsalzgehalt des Blutes, die Ausscheidung des $\mathrm{NaCl}$ in dem diuretischen Harn dar? - In den Versuchen mit NaCl-Einlauf stieg z. B. der NaCl-Gehalt des Blutes von 0,655 auf 0,780 Proc.: Der Harn enthielt 1,18 Proc. Kochsalz. - In einem Glaubersalzversuche (Nr. 4) stieg der $\mathrm{Na}_{2} \mathrm{SO}_{4}$-Gehalt, während der $\mathrm{NaCl}$-Gehalt von 0,632 a uf 0,600 sank. Der Harn enthielt über 2 Proc. Glaubersalz und war fast kochsalzfrei. Während des Einlaufs wurde ein Urin

1) Hamburger, Zeitschr. f. Biol. Bd. XXVII. S. 259. 1890. 
von 0,067 Proc., nach Schluss desselben von 0,050 Proc $\mathrm{NaCl}$ gesammelt. - In einem anderen Versuche (Nr.6) waren die Kochsalzconcentrationen des Glaubersalzharnes 0,067 und 0,073 Proc. (gegen 2,45 und 3,56 Proc. $\mathrm{Na}_{2} \mathrm{SO}_{4}$ ). Die Niere macht demnach aus einem Blut, welches 0,6 Proc. $\mathrm{NaCl}$ und nur 0,27 Proc. $\mathrm{Na}_{2} \mathrm{SO}_{4}$ enthält, einen fast kochsalzfreien, aber sehr stark glaubersalzhaltigen Harn. Sie bestreitet ihren Salzbedarf für den diuretischen Harn nicht wahllos aus dem Gesammtsalzbestande des Blutes, sondern besitzt deutliche Auswahlsfähigkeit. Sie reagirt sehr stark auf die Aenderung des Gehaltes an einem einzelnen Blutbestandtheil, einerlei ob derselbe zu den in grösserer oder kleinerer Menge im Blute enthaltenen zählt. Die Niere functionirt also nicht nur für den Zucker, wie dies $M \mathrm{unk}^{1}$ ) gezeigt hat, als ein Ueberlaufsventil, das nur in Thätigkeit tritt, wenn eine bestimmte Concentrationssehwelle im Blut überschritten wird, sondern auch für jedes einzelne $\mathrm{Salz}$ (zum mindesten für $\mathrm{NaCl}$ und $\mathrm{Na}_{2} \mathrm{SO}_{4}$ ). Steigt dessen Gehalt im Blut, so erfolgt Ausscheidung, sinkt er unter eine gewisse Grenze, so wird das Salz durch die Niere so gut wie gar nieht ausgeschieden ${ }^{2}$.

Durch die angeführten Befunde erhalten die Untersuehungen v. Lim becks, die ihn ebenfalls zur Annahme einer Secretionsschwelle für die einzelnen Salze führten, eine thatsächliche Bestätigung: Weiter steht augenscheinlich hiermit in Zusammenhang, dass im Chlorhunger nach einigen Tagen, wenn der Organismus an Chloriden verarmt ist, der Harn fast kochsalzfrei wird, und dass ein gewisses Minimum ron Kochsalz mit der grössten Zähigkeit im Körper zurïckgehalten wird ${ }^{3}$ ).

Man sieht also, dass die Niere an der Abnahme des proc. NaClGehaltes des Blutes nicht nur unbetheiligt ist, sondern ihr sogar entgegen zu wirken strebt, indem sie fast kochsalzfreios Wasser

1) Munk, Virchow's Archiv Bd. CVII. S. 291. 1887.

2) In der I. Mittheilung sind Fälle beschrieben, in denen vach Infusion bypotonischer Lösungen (z. B. Versuch 9 S. 114) der procent. Gehalt des Serums an allen festen Bestandtheilen, also auch des Kochsalzes, sank, und trotzdem ein Harn von 0,8-1 Proc. NaCl entleert wurde. Hier handelt es sich jedoch um eine reine Wasserdiurese, die Niere arbeitet, weil das Blut stark verdünnt ist und nimmt jetzt von dem im Blut relativ am reichlichsten vertretenen Salze, dem NaCl, mit in den Harn, da sie sonst destillirtes Wasser ausscheiden müsste, wozu eine enorme osmotische Arbeit gehörte (vgl. Dres.er, Archiv f. experiment. Pathol. u. Pharmakol. Bd. XXIX. S. 303. 1892). In den hier oben geschilderten Versuchen dagegen ist der Harn schon durch das Glaubersalz concentrirt genug, so dass dieser Zwang zur NaCl-Ausscheidung nicht besteht.

3) Cahn, Zeitschr. f. pbysiolog. Chemie Bd. X. S. 522. 1856. 
dem Blute entzieht. Sie arbeitet hier also in entgegengesetztem Sinne wie der NaCl-Austausch durch die Gefässwände. Letzterer bewirlit, wie Hamburger betonte, dass der osmotische Druck des Blutes nicht zu stark nach $\mathrm{Na}_{2} \mathrm{SO}_{4}$-Einfuhr steigen kann. Es wirkt demnach unter diesen Umständen die Niere der "Regelung des wasseranziehenden Vermögens des Blutes" entgegen.

Es bat sich ergeben, dass die stärkere diuretische Wirkung des Glaubersalzes nicht nur nicht auf einer stärkeren Blutverdünnung, sondern auch nicht auf einem stärkeren Salzgehalt (Gesammtsalzgehalt und Gehalt an dem betr. einzelnen Salze) des Blutes beruht. Es musste nun noch untersucht werden, ob etwa die mechanischen Bedingungen, die Kreislaufsverhältnisse, sich nach Glaubersalzeinfubr anders verhalten als nach $\mathrm{NaCl}-$ Infusion.

\section{Die Kreislaufsverhältnisse bei $\mathrm{NaCl}$ - und $\mathrm{Na}_{2} \mathrm{SO}_{4}$ - Infusion.}

Dass die Salzdiurese nicht bedingt sein kann durch Aenderungen des arteriellen Druckes, ist durch vielfältige Beobachtungen erwiesen. Sie tritt ebensogut bei gleich hohem wie auch bei gesunkenem Carotidendruck ein. Dagegen hat neuerdings Starling ${ }^{1}$ ) versucht, auch in dieser Richtung den alten Ludwig'schen Lehren durch neue Versuche und Ueberlegungen eine Stïtze zu geben. Sein Gedankengang ist etwa folgender: Es ist unrichtig, aus dem arteriellen Blutdruck irgend welche Schlüsse auf den Capillardruck (hier also in den Glomerulis) machen zu wollen. Vielmehr wird sich der Capillardruck eher den Schwankungen des Venendrucks anschliessen. Speciell wird bei gleichem arteriellen Druck der Capillardruck dann steigen, wenn der Venendruck sich stark hebt. Dieses gilt stricte nur, solange sich die peripheren Kreislaufswiderstände in den kleinen Arterien, die also v or den Capillaren eingeschaltet sind, nicht ändern. Wird der periphere Widerstand durch Erweiterung der Arteriolen in einem begrenzten Gefässgebiet herabgesetzt, so muss bei gleichbleibendem arteriellen und venösen Druck sich der Capillardruck in diesem Gebiet ebenfalls heben. Beides zusammen also, Steigerung des venösen Druckes und Vasodilatation eines bestimmten Gefässbezirkes wird bei gleichem arteriellen Druck eine starke Steigerung des Capillardrucks herbeifübren.

Dieses soll nun nach Starling in der Niere bei der Salzdiurese verwirklicht sein: Nach Injection einer starken Salz- oder Zucker-

1) Starling, Schäfer's Text-book of physiol. Vol. I. p. 647. 1898. - Journa of physiol. Vol. XXIV. p. 317. 1899 und Vol. XVI. p. 159. 1894. 
lösung kommt es zur Blutverdünnung, zur hydrämisehen Plethora. Während die Aenderungen des arteriellen Druekes gering sind, steigt der Venendruck alsbald hoch an. Folglich muss auch der Capillardruck sich heben. Zweitens erfolgt active Vasodilatation in den Nierengefässen, die sich durch eine Zunahme des Nierenvolums bezw. durch einen Ausschlag des Onkometers zu erkennen giebt. Der Erfolg muss ebenfalls eine Zunahme des Capillardrucks in der Niere sein. In Folge dieses gesteigerten Filtrationsdrucks im Glomerulus (verbunden mit zunehmender Strömungsgesehwindigkeit in demselben), und nicht in Folge der Aenderung der Blutbeschaffenheit soll dann die Diurese eintreten.

Stellt man sich zunächst auf den Boden dieser Anschaung, so ergeben sich für die vorliegende Untersuchung folgende Fragen:

1. Führt vielleicht Glaubersalz eine stärkere Steigerung des venösen Druckes herbei als das Kochsalz, trotzdem beide gleiche hydrämische Plethora bedingen?

2. Ist die active Vasodilatation in der Niere bezw. der Onkometerausschlag bei $\mathrm{Na}_{2} \mathrm{SO}_{4}$-Infusion grösser als nach Einfuhr von $\mathrm{NaCl}$ ?

Zur Entscheidung der ersten Frage wurden Versuche an Hunden angestellt. Die Versuchsbedingungen waren im Wesentlichen die gleichen, wie sie bei den oben geschilderten Experimenten eingehalten wurden. Die Druckbestimmungen wurden nach Starling's Angaben ausgeführt.

Ein Theil der Hunde wurde direct aus dem Stall, ein anderer Theil nach 4 tägigem Hunger verwendet. In tiefer Morphinnarkose wurde das Thier ans Kymographion gebracht, der arterielle Druck von der Art. femoralis aus alle $\mathbf{5}$ Minuten registrirt. Der Venendruck (Seitendruck der Vena cava) wurde von der Vena femoralis aus gemessen. Das betr. Manometer war mit $\mathrm{MgSO}_{4}$ (25 Proc.) gefüllt ${ }^{1}$ ), der Stand der Flüssigkeitssäule wurde alle 5 Minuten gleichzeitig mit dem arteriellen Druck abgelesen und notirt. Der Harn tropfte aus Ureterenkanülen und wurde alle 10 Minuten gemessen. Nachdem die Manometer und die Secretion constante Werthe angenommen hatten, lief wieder 50 Minuten lang eine der beiden Salzlösungen mit der Einlaufsgeschwindigkeit von $0,2 \mathrm{cem}$ pro Minute und Kilogramm in die Jugularis.

1) Die Werthe sind stets in Höhen der 25 procent. $\mathrm{MgSO}_{4}$-Säule angegeben. Man muss sie also durch 13 dividiren, um auf Quecksilberwerthe zu kommen. 
Das Resultat war in allen Versuchen dasselbe. Es $\mathbf{k}$ a $\mathrm{m}$ überhapt nichtzueiner wesentlichen Steigerung des Venendruckes. Zur Illustration seien 2 Versuche in Curvenform angeführt. Die obere Linie entspricht dem arteriellen Druck, die mittlere starke dem Venendruck, die untere punktirte der Diurese.

Curve 4.

Hund 8 , hat nicht gehungert. Einlauf von $\mathrm{Na}_{2} \mathrm{SO}_{4}$

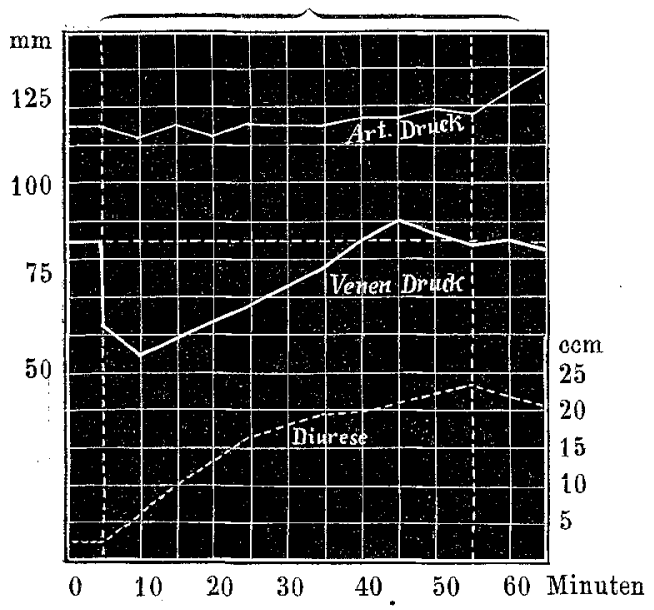

Man sieht, dass der arterielle Druck sich während des Einlaufs überhaupt nieht ändert. Der venöse Druck dagegen sinkt mit Beginn der Infusion sofort von 85 auf 61 und $55 \mathrm{~mm}$ und hebt sich darauf langsam wieder, um seinen normalen Werth erst nach 40 Minuten zu erreichen und ihn dann um $5 \mathrm{~mm}$ Wasser zu übersteigen. Die Diuresse dagegen erhebt sich sofort nach Beginn des Einlaufs, steigt in den ersten beiden Perioden stark, und nachher langsam an. Hier hat also bei gesunkenem Venendruck Salzdiurese stattgefunden.

(Curve 5 siehe folgende Seite.)

Hier seben wir eine starke Diurese eintreten, ohne dass sich der Venendruck wesentlich ändert (er fällt um $7 \mathrm{~mm}$ ). Auch der arterielle Druck bleibt in den ersten 40 Minuten, bei schon bestehender starker Harnflut, niedrig und hebt sich erst zuletzt bis auf $150 \mathrm{~mm}$, ohne dass dabei die Diurese mit steigt.

Diese Versuche, deren Ausfall mir selbst überraschend war, zeigen also, dass die Injection von starken Salzlösungen, wenn sie nur hinreichend langsam geschieht, trotz der entstehenden hydrämischen Plethora nicht den Venendruck zu steigern braucht, dass also 
die alte angefochtene Angabe von Cohnheim und Lichtheimi) za Recht besteht. Weiter zeigt sich, dass starke Salzdiuresen auftreten können, ohne dass der Venendruck steigt, ja sogar, wenn er stark sinkt. Die Ursache, dsss Starling gegentheilige Resultate erhielt, liegt vielleicht darin, dass er die intravenösen Injectionen sehr schnell vornahm. So wurden in einem Versuch ${ }^{2}$ ) $40 \mathrm{~g}$ Dextrose in 5 Minuten injicirt. In unseren Experimenten wurde 50 Minuten lang nur je $0,2 \mathrm{ocm}$ pro Minute und Kilogramm eingeführt.

Da sich herausstellte, dass Steigerung des Venendrucks für die Salzdiurese in den hier geschilderten Versuchen irrelevant ist, konnten natürlich Unterschiede zwischen $\mathrm{NaCl}$ - und $\mathrm{Na}_{2} \mathrm{SO}_{4}$-Einfuhr sich nicht ergeben.

Curve 5.

Hund 9 hungert 4 Tage. Einlauf ron $\mathrm{NaCl}$.

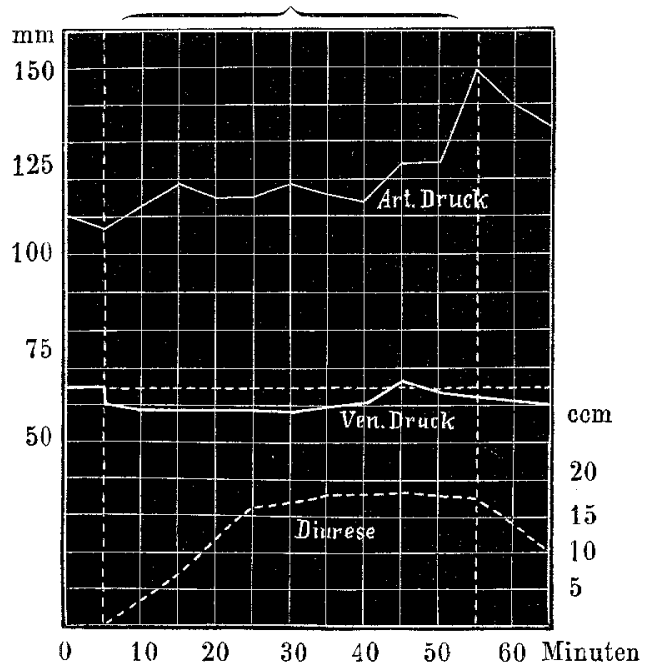

Nachdem so ermittelt war, dass Aenderungen des allgemeinen Kreislaufs für die stärkere Diurese nach Glaubersalzeinfuhr nicht verantwortlich gemacht werden konnten, fragte es sich weiter, wie der locale Blutlauf durch die secernirende Niere sich bei NaClund $\mathrm{Na}_{2} \mathrm{SO}_{4}$-Infusion verhält. Da wir leider die Blutgeschwindigkeit in der Nierenarterie oder-Vene während der Thätigkeit des Organes im Thiere noch nicht einwandsfrei messen können, so bleibt nur

1) Cohnheim und Lichtheim, Virch. Arch. Bd. LXIX. 1877. S. 106.Ges. Abhandl. S. 556.

2) Schäfer's Text-book of Physiol. Vol. I. p. 294. 1898. 
übrig, die Ausschläge des Onkometers zu registriren und aus den Volumensänderungen des Organs auf die Grösse der Durchblutung zu schliessen. Solche Versuche sind von Cohnheim und Roy ${ }^{1}$ ) und neuerdings von Starling gemacht; dieser behauptete die Abhängigkeit der Diurese von der Grösse der Durchblutung. Eine grosse Anzahl von Onkometerversuchen, die von Herrn Prof. Gottlieb und mir mit verschiedenen Diureticis angestellt wurden und die demnächst in diesem Archiv veröffentlicht werden sollen, haben uns die Ueberzeugung beigebracht, dass der gesteigerte Blutfluss durch die Niere nur eine Begleiterscheinung, nicht aber die Ursache der Diurese ist. Die Onkometrie bei $\mathrm{NaCl}$ - und $\mathrm{Na}_{2} \mathrm{SO}_{4}$-Infusion hat diese Ansicht bestätigt.

Auch diese Versuche wurden an Kaninchen angestellt, die 4 Tage gedurstet und die 2 letzten Tage Hafer erhalten hatten. Es wurde arterieller Blutdruck, Onkometerstand und die Harnmenge jeder Niere fiur sich gemessen. Die Infusion dauerte wieder $50 \mathrm{Mi}$ nuten mit einer Einlaufsgeschwindigkeit von $0,5 \mathrm{ccm}$ pro Minute und Kilogramm. Die Thiere waren mit Urethan (1,0 pro Kilogramm per os) in 10 procent. Lösung narkotisirt. Die genaue Technik der Onkometrie und die dabei beobachteten Cautelen werden in der erwähnten Arbeit,ausführlich mitgetheilt werden, so dass hier darauf verwiesen werden kann.

Das Ergebniss war, dass bei beiden Salzen in der Regel ein Steigen des Onkometerstandes erfolgte, dass aber eine stärkere Volumenszunahme der Niere bei der Glaubersalzdiurese nicht zu beobachten war. Im Gegentheil schien Kochsalz eine grössere Expansion des Organs bewirken zu können als Glaubersalz. Die maximale Volumenszunahme während der Diurese betrug nach $\mathrm{NaCl} 12$ bis 18 Proc., nach $\mathrm{Na}_{2} \mathrm{SO}_{4}$ nur 2,4-7,5 Proc. des Nierenvolums. Das Gesagte mag dureh einige Beispiele in Curvenform illustrirt werden.

Die starke Linie giebt den Onkometerausschlag in Procent des Nierenvolumens an. Wenn z. B. am Schluss des Versuchs die Linie auf 4,9 steht, so heisst das, dass das Nierenvolumen um 4,9 Proc. zugenommen hat. Es ist dies also ein absoluter und vergleichbarer Werth. Die feine Linie giebt die Diurese in Cubikcentimeter pro Kilogramm Thier (also ebenfalls vergleichbare Werthe) an, und zwar nur die Secretion aus der onkometrirten Niere. Die punktirte Linie entspricht dem Blutdruek. Man sieht, dass das Onkometer nach 
vorübergehendem Sinken ${ }^{1}$ ) sich uber die - punktirte - O-Linie erhebt, schnell seinen höchsten Stand erreicht und diesen bis zum Schluss des Versuches beibehält. Die Diurese dagegen ist zur Zeit, wo das Onlsometer schon seinen hohen Stand erreicht hat, noch niedrig. Dagegen erhebt sie sich nachher stark, während das Onkometer seinen Stand beibehält und sinkt nach Schluss des Einlaufs rapide, während das Nierenvolumen sich kaum ändert. Also: Erhebung des Onkometers bei geringer Diurese, stark steigende und schnell sinkende Diurese bei gleichem Onkometerstande.

Curve 6.

Kaninchen Nr. 25. Einlauf von $\mathrm{Na}_{2} \mathrm{SO}_{4}$.

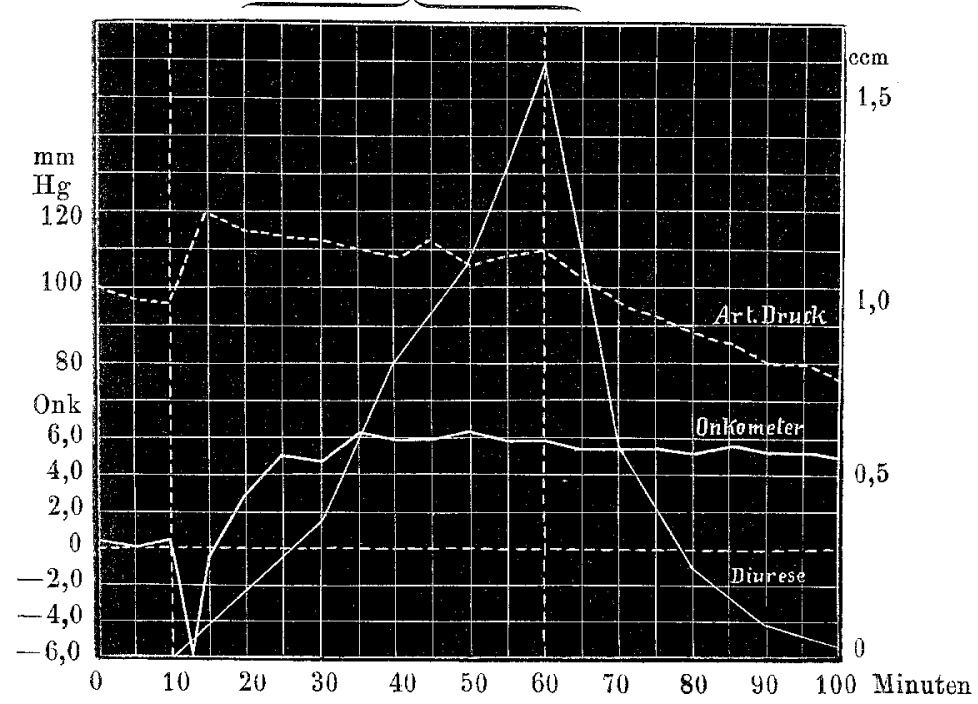

Zum Vergleich sei jetzt (s. Curve auf folgender Seite) ein Versuch mit $\mathrm{NaCl}$-Infusion angeführt.

Hier hebt sich ebenfalls das Onkometer schnell und erreicht nach 25 Minuten seinen höchsten Werth, um dann noch während des Einlaufs wieder zu sinken. Nach Schluss der Infusion erfolgt rapider Abfall. Man sieht, dass die maximale Volumenszunahme hier 18 Proc. betrug, gegen 6 Proc. im vorherigen $\mathrm{Na}_{2} \mathrm{SO}_{4}$-Versuch. Trotzdem erhebt sich die Diurese hier viel weniger hoch. Auch hier sehen wir, dass die Harnsecretion noch niedrig ist, während das Onkometer schon den höchsten Stand erreicht hat, und dass sie

1) Dieses Sinken des Onlrometers ist eine Ausnabme. In den meisten Fällen steigt das Nierenvolumen direct nach Beginn des Einlaufs. 
dann sich noch um mebr als das doppelte erhebt bei sinkendem Onkometerstande (von 35-50 Minuten). Nach Schluss des Einlaufs fallen Onkometer und Diurese parallel.

Curve 7.

Kaninchen Nr. 26. NaCl-Infusion,

Einlauf von $\mathrm{NaCl}$.

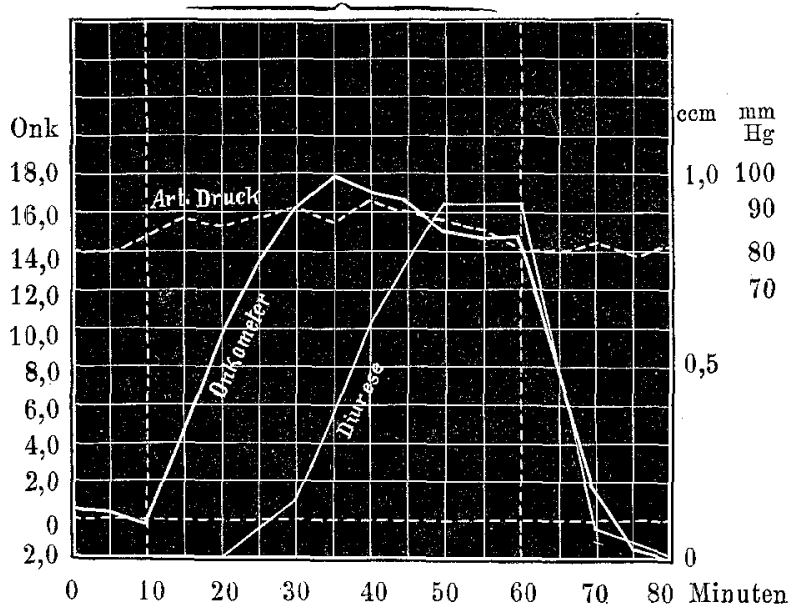

Wie unabhängig aber diese ganze Salzdiurese vom Onkometerstand sein kann, erhellt aus dem nachstehenden Beispiel:

Curve 8.

Kaninchen Nr. 29. $\mathrm{Na}_{2} \mathrm{SO}_{4}$-Infusion.

Einlauf von $\mathrm{Na}_{2} \mathrm{SO}_{4}$

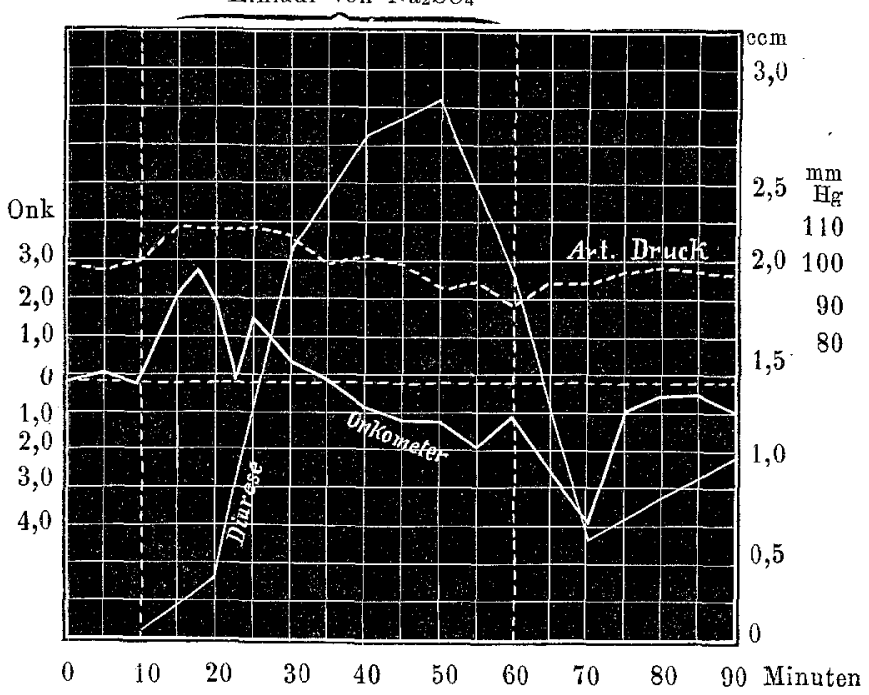


Hier steigt das Onkometer nur wälrend der ersten 7 Minuten des Einlaufs, um dann bis nach Schluss desselben continuirlich zu sinken. Die Diurese aber erhebt sich zu beträchtlicher Höhe, während das Nierenvolumen sinkt. Ja, noch mehr: während der zweiten Hälfte der Infusion ist das Nierenvolumen geringer als vor Beginn des Versuchs, und in diese Zeit des negativen Onkometerstandes fällt das Maximum der Diurese.

Es lässt sich nicht sinnfälliger zeigen, dass ein Parallelismus zwischen Nierenvolumen und Diurese nicht besteht, dass die $\mathrm{Na}_{2} \mathrm{SO}_{4}$ Diurese nicht von stärkerer Nierendurchblutung abhängt, ja, dass sie ohne Steigerung des Onkometerstandes eintreten kann. 1)

Die Untersuchung der Kreislaufsverhältnisse bei der Infusion der beiden Salze hat gezeigt, dass weder eine stärkere Steigerung des Venendrucks, noch eine grössere Expansion der thätigen Niere mit consecutiver stärkerer Durchblutung die Ursache der nach $\mathrm{Na}_{2} \mathrm{SO}_{4}$ auftretenden grösseren Harnfluth sein kann. Erinnern wir uns der im Anfang dieses Abschnittes erwälınten Ausfïhrung Starling's, so kann man sagen, dass eine höhere Steigerung des Capillardrucks nach Glaubersalzeinlauf nicht eintritt, als nach Kochsalzzufuhr.

Weiter hat sich aber herausgestellt, und dieses ist das Wichtigere, dass es bei der Salzdiurese weder zu einer Steigerung des Venendrucks, noch zu einer der Diurese parallelen Steigerung des Nierenvolumens zu kommen braucht. Dass bei gleich bleibendem Venendruck und bei gleichem oder sinkendem Onkometerstande die Harnfluth schwellen kann, dass mit anderen Worten auch ohne Steigerung des Capillardrucks (im Sinne von Starling) eine Salzdiurese eintreten kann. Daraus ergiebt sich, dass die Steigerung des Capillardruoks das primum movens für die Salzdiurese nicht sein kann.

Man wird daher auch dem Capillardruck nicht die Rolle der ausschliesslich treibenden Kraft für die normale Harnsecretion zusprechen können.

\section{Schlussfolgerungen.}

Es hat sich herausgestellt, dass eine Glaubersalzlösung eine viel stärkere diuretische Wirkung entfaltet, als eine ihr isotonische Kochsalzlösung. Die Untersuchung der einzelnen hierbei in Betracht kommenden Factoren, ergab, dass diese stärkere Diurese nicht be-

1) Dass die Diurese nicht mit dem arteriellen Blutdruck parallel geht, ergiebt die einfache Inspection der 3 Curven. Auf die Abhängigkeit des Onkometerstandes vom arteriellen Blutdruck wird a. a. 0 . eingegangen werden. 
ruht auf einer grösseren Blutverdünnung, dass vielmehr beide isotonischen Flüssigkeiten gleiche Blutverdünnung hervorrufen; dass sie weiter nicht beruht auf einer Zunahme des Gesammtsalzgehaltes im Blute, sondern dass dieser nach $\mathrm{Na}_{2} \mathrm{SO}_{4}$-Infusion sogar niedriger ist; dass sie nicht darauf beruht, dass von dem diuretisch wirksamen Salze ein grösserer Bruchtheil im Blute kreist, denn von dem eingeführten $\mathrm{NaCl}$ ist sogar etwas mehr in der Blutbahn als vom $\mathrm{Na}_{2} \mathrm{SO}_{4}$. Auch eine Steigerung des Venendrucks kann nicht verantwortlich gemacht werden, denn die Diurese tritt in beiden Fällen auch ohne dieselbe ein. Das heisst mit anderen Worten: die ausserhalb der Niere sich abspielenden Vorgänge sind die gleichen nach Zufuhr der beiden Salze. Es musste demnach der Angriffspunkt der diuretischen Wirkung in die Niere selbst verlegt werden. Hier liess sich zeigen, dass ebensowenig die active Vasodilatation der Nierengefässe für die stärkere Diurese nach Glaubersalz verantwortlich gemacht werden kann, sondern dass eher nach $\mathrm{NaCl}$ der Blutfluss durch die Niere grösser ist, wie denn überhaupt ein directer Parallelismus zwisehen Harnfluth und Blutstrom durch die Niere nicht besteht. Aus den beiden letzten Versuchsreihen zusammen folgt, dass auch die Steigerung des Capillardrucks in der Niere nicht für die stärkere Glaubersalzdiurese angeschuldigt werden kann.

Es bleibt also nur noch eine Einwirkung auf die secernirenden Elemente der Niere selbst übrig, die wir zunächst kurz in der Weise beschreiben können, dass die thätige Niere in der Zeiteinheit relativ mehr Glaubersalz aus dem Blute fortschafft als Kochsalz. Nähere Fingerzeige geben die Resultate, die über die Abhängigkeit der Salzconcentration des Harnes von der des Blutes erhalten wurden. Für Kochsalz zeigte sich, dass der NaCl-Gehalt des Blutes nur um ein Weniges zu steigen braucht, damit ein sehr kochsalzreicher Harn ausgeschieden wird. Andererseits wird bei sinkendem $\mathrm{NaCl}-$ Gehalt im Blut der Urin fast kochsalzfrei. Unter diesen Umständen tritt aber ein Salz, von dem proc. viel weniger im Blute enthalten ist als vom Kochsalz, nämlich das Glanbersalz, sofern nur sein Gehalt im Blute gesteigert ist, in grosser Menge durch die Niere. Man muss also schliessen, dass die Niere eingestellt ist auf eine bestimmte Zusammensetzung des Blutes, und dass sie, sobald ein einzelner Bestandtheil des Blutes erhöht ist, diesen beseitigt. In der I. Mittheilung ist ausführlieh gezeigt worden, dass dieses vor Allem für das Wasser gilt, dass man die hochgradigsten Diuresen erhält bei gesunkenem Salzgehalt, aber gesteigertem Wassergehalt des Blutes. Für die einzelnen Salze gilt dieses ebenso gut. Da nun 
die Niere weder Wasser ohne Salz, noch Salz ohne Wasser ausscheiden kann, so ist das Resultat für die Zusammensetzung des Körpers nach Ablauf der Diurese nicht gleichgültig. Es wird bei der Salzdiurese dem Körper Wasser, und bei der Wasserdiurese Salz entzogen. Es ist also mit dem Inkrafttreten dieser Schutzvorrichtung eine gewisse Unzweckmässigkeit für den Organismus verbunden. Der Vergleich der Grösse der Salzausscheidung mit der des Wassers hat ergeben, dass im Mittel umsomehr Wasser fortgeht, je mehr Salz ausgeschieden wird. Es muss also an eine enge Beziehung zwischen Salz- und Wasserbewegung durch die Niere gedacht werden. Ueber die nähere Art dieses Zusammenhanges geben unsere Versuche keinen Aufschluss. $O b$ es sich um einfaches Zusammengehen beider Ausscheidungen handelt, oder $o b$ in Folge der Blutrerdünnung Wasserdiurese, und dazu noch in Folge der Zunahme eines Salzes im Blute Secretion dieses Salzes mit einer zweiten Menge Wassers stattfindet, oder ob die Dinge noch verwickelter liegen, muss vorläufig unentschieden bleiben.

Es giebt demnach, wenn man die Blutbeschaffenheit ins Auge fasst:

a) reine Wasserdiuresen (I. Mittheilung), d. h. Diuresen, bei denen nur der Wassergehalt des Blutes erhöht ist;

b) combinirte Wasser- und Salzdiuresen. Dieses sind alle Diuresen nach intravenöser Zufuhr starker Salzlösungen. Hier wirken Blutverdünnung und Zunahme eines Salzes im Blute zusammen.

c) reine Salzdiuresen. Diese stellen sich z. B. regelmässig am Ende der Glaubersalzversuche ein, wenn die Blutverdünnung zurückgegangen, das Blut sogar wasserärmer geworden ist, und nur noch die Vermehrung des $\mathrm{Na}_{2} \mathrm{SO}_{4}$ die Niere zur Thätigkeit zwingt.

Nach allem Vorhergehenden muss man also den grössten Werth auf die Aenderung der Blutbeschaffenheit) für den Eintritt der Diurese legen und nicht auf die Kreislaufsverhältnisse. Wenigstens für die combinirte Salz- und Wasserdiurese und die reine Salzdiurese ist der Nachweis geführt, dass sie unabbängig von Zunahme des Venendrucks, Onkometerstandes und Capillardrucks bestehen und zunehmen können.

1) Ebenso wie in der ersten Mittbeilung bereits nachgewiesen wurde, dass der osmotische Druck des Blutes nicht das Wesentlichste für die Diurese sein kann, zeigt sich auch hier wieder, dass der stärkeren Diurese der geringe osmotische Druck entspricht, worauf in Hinblick auf manche neuere Anschauungen besonders hingewiesen werden soll. 
Es müssen also Einrichtungen in der Niere sein, die auf Veränderung der Blntbeschaffenheit mit gesteigerter Thätigkeit reagiren. Die Ludwig'sche Filtrationstheorie scheint mir auch in ihrer neuen Gestalt die Thatsachen nicht in einfacher Weise erklären zu können.

Im Gegensatz zur Niere zeigen die Gefässwände, durch welche hindurch der Austausch zwischen Blut und Gewebe stattfindet, keine wesentlichen Unterschiede der Durchlässigkeit beiden Salzen gegenüber. Die Vertheilung von $\mathrm{NaCl}$ und $\mathrm{Na}_{2} \mathrm{SO}_{4}$ zwischen jenen beiden findet ungefähr in gleichen Verhältnissen statt, und die gleichen Mengen des in der Blutbahn kreisenden Salzes bedingen gleiche Blutverdünnung. Ueberhaupt unterliegt der Wasser- und Salzstrom durch die Gefässwände nach hochgradigen Aenderungen des Salz- und Wassergleichgewichtes, wie er durch derartige Infusionen statt hat, im Allgemeinen den Regeln der osmotischen Druckdifferenzen; so lange diese noch hochgradig vorhanden sind, kann man einen richtenden Einfluss der Capillarwände nicht nachweisen. Erst wenn sie sich mehr oder weniger ausgeglichen haben, scheint eine solche Thätigkeit sich geltend zu machen, mit dem Resultat, dass die Gewebe als Depots für überschüssiges Wasser und Salz functioniren müssen, wäbrend das Blut seine alte Zusammensetzung wieder bekommt. (Vgl. dazu I. Mittheilung S. 100).

Die Ergebnisse der Untersuchung lassen sich in folgenden Sätzen zusammenfassen:

1. Der Vergleich der diuretischen Wirkungen isotonischer Lösungen von $\mathrm{NaCl}$ und $\mathrm{Na}_{2} \mathrm{SO}_{4}$ unter gleichen Bedingungen der intravenösen Injection ergiebt das Resultat, dass Glaubersalz bei Kaninchen und Hunden fast doppelt so stark diuretisch wirlat als Kochsalz. Das Glaubersalz erweist sich dabei auch als fast doppelt so harnfähig.

2. Analysirt man die einzelnen für die Diurese in Betracht kommenden Factoren, so ergiebt sich, dass der Grund der stärkeren diuretischen Wirksamkeit des Glaubersalzes in keiner der ausserhalb der Niere selbst gelegenen Bedingungen gefunden werden kann, denn weder ruft Glaubersalz eine stärkere Blutverdünnung hervor als Kochsalz, noch kreist ein grösserer Bruchtheil von dem eingeführten Salze in der Blutbahn. Ebenso wenig bieten die Kreislaufsverhältnisse Unterschiede dar. Vielmehr ruft $\mathrm{Na}_{2} \mathrm{SO}_{4}$ bei gleicher Blutverdïnnung, bei gleicher Vertheilung und bei gleichem Capillardruck die stärkere Diurese hervor. 
3. Lässt sich somit in den genannten Bedingungen die Ursache der verschiedenen Wirksamkeit der beiden Salze nicht finden, so muss der Angriffspunkt der verschiedenen Wirkung in den secernirenden Elementen der Niere selbst gesucht werden.

4. Alle Ergebnisse der vorliegenden Arbeit führen zu der Annahme, dass sowohl für das Wasser als auch für die einzelnen Salze im Blute eine Secretionsschwelle besteht, deren Ueberschreitung den Eintritt der Diurese zur Folge hat. Schon die Blutverdünnung allein kann Diurese erzeugen - Wasserdiurese -, andererseits kann die alleinige Zunahme eines Salzes im Blute Harnfluth hervorrufen - Salzdiurese. Bei der intravenösen Injection starker Salzlösungen wirken diese beiden Momente zusammen - combinirte Salz- und Wasserdiurese.

\section{Analytisches Verfahren.}

Zur Bestimmung der Gefrierpunktserniedrigung diente der Beckmann'sche Apparat. Kochsalz im Harn wurde nach Salkowski') mit $\mathrm{AgNO}_{3}$ und $\mathrm{CNSNH}_{4}$ titrirt. Zur Bestimmung des $\mathrm{NaCl}$ im Serum wurde dieses nach Bunge und Behaghel v. Adlerskron ${ }^{2}$ ) unter Zusatz von chlorfreier Soda eingedampft und verascht und dann ebenfalls nach Salkowski titrirt.

Glaubersalz im Harn wurde durch Ausfällen der Schwefelsäure mit Salkowski'scher Barytmischung: unter Essigsäurezusatz, Veraschen und Wägen bestimmt.

Bei der quantitativen Ermittelung der Sulfate im Serum ist es nicht angängig, dieses zu veraschen, da dann der Schwefel der Eiweisskörper als $\mathrm{H}_{2} \mathrm{SO}_{4}$ mitbestimmt wird. Es wurde deshalb aus $10 \mathrm{ccm}$ Serum das Eiweiss durch Hitze anskoagolirt, abfiltrirt und sehr sorgfältig bis zum Verschwinden der $\mathrm{Cl}$ - und $\mathrm{H}_{2} \mathrm{SO}_{4}$-Reaction ausgewaschen; im Filtrat dann die Schwefelsäure mit $\mathrm{HCl}$ und $\mathrm{BaCl}_{2}$ gefällt, nach allgemeinen Regeln verascht und gewogen. Zwei Controlbestimmungen ergaben auf diese Weise:

$$
\begin{aligned}
& 0,0035 \\
& 0,0037 \\
& 0,0036 \mathrm{BaSO}_{4}=0,0022 \mathrm{Na}_{2} \mathrm{SO}_{4}=0,022 \text { Proc. }
\end{aligned}
$$

Zugesetztes Glaubersalz lässt sich nach diesem Verfahren ziemlich vollständig wiedergewinnen:

1) Salkowski, Centr. f. d. Med. Wiss. Bd. XIX. S. 10. 1881.

2) v. Adlerskron, Zeitschr. f. analyt. Chemie Bd. XII. S. 390. 1873. 


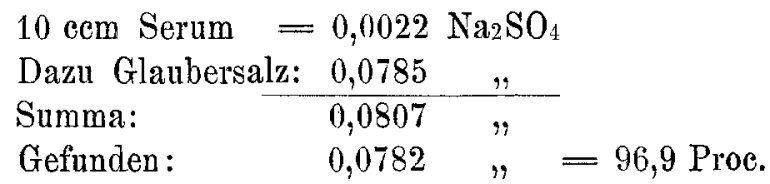

Es lässt sich demnach die Sulfatbestimmung im Serum auf die greschilderte Weise mit für die Versuche hinreichender Genauigkeit ausführen.

Im Darminhalt wurden vor der Sulfatbestimmung alle eiweissartigen Substanzen mit $\mathrm{HCl}$ und Wismuthjodidjodkalium ausgefällt, abfiltrirt und sorgfältig ausgewaschen.

Für die Hämoglobinbestimmung wurde. wieder das Hämoglobinometer von Miescher und Veillon ${ }^{1}$ ) benutzt, das sich nach unseren Erfahrungen durch grosse Genauigkeit auszeichnet. $\mathrm{Zu}$ dem entgegengesetzten Resultat ist allerdings A. Loew ${ }^{2}$ ) gekommen. $\mathrm{Er}$ fand, dass mit abnehmender Schichtdicke und abnehmender Concentration der Hb-Lösung die Ergebnisse zu niedrig ausfielen. So erhielt er

bei einer Schicht von $15 \mathrm{~mm}$ : 5,9 Proc. $\mathrm{Hb}$, and in einem anderen Versuch " $12,: 3,9$ " " bei einer Verdiunnung 1:200:5,76 Proc. Hb, " " $\quad 1: 300: 4,95, "$, . Im letzteren Falle ermittelte er den wahren Gehalt der Lösung durch Trockenbestimmung zu 6,61 Proc. Hb.

Ich halte es für wahrscheinlich, dass das von Loew y benutzte Instrument ein zufällig besonders mangelhaftes war, eine Möglichkeit, die er selbst schon ins Auge fasste. Wenigstens babe ich sowohl wie Andere, die mit unserem Exemplar arbeiteten, weit bessere Resultate erzielt.

Es wurde stets im Dunkelzimmer hinter einem schwarzen Pappschirm gearbeitet, der den Gasbrenner für das Auge verdeekte. Auf gleiche Flammenhöhe und gute Dunkeladaptation des Auges sowie auf die zahlreichen von Veillon angegebenen Cautelen wurde geachtet. Jede Blutprobe wurde bei einer Schicht von 12 und $15 \mathrm{~mm}$ untersucht und jedesmal 10, also im Ganzen 20 Ablesungen gemacht. Nie waren so grosse Abweichungen zu constatiren, wie diesLoew y fand.

Für die Genauigkeit der mittleren Region des Glaskeils wurde eine besondere Bestimmung mit je 20 Ablesungen ausgeführt:

1) Veillon, Archiv f. exper. Pathol. u. Pharmakol. Bd. XXXlX. S. 385.1897.

2) A. Loewy, Centr. f. Med. Wiss. Bd. XXIX. S. 497. 1898. 
I. Erste Mischpipette: (Verdünnung: 0,9925:300)

Schichtdicke $15 \mathrm{~mm} 20$ Ablesungen: $63,875=16,07$ Proc. Hb

$$
\begin{aligned}
& 12 \text { "Mittel: } 16,085 \text { Proo. Hb. } \\
& \text { II. Zweite Mischpipette: (Verdünnung: } 0,97: 400 \text { ). }
\end{aligned}
$$

Sehichtdicke $15 \mathrm{~mm} 20$ Ablesungen: $47,325=16,26$ Proc. $\mathrm{Hb}$

$$
\text { " } 12 \text { "Mittel: } 16,100 \text { Proc. } \mathrm{Hb} \text {. } 37,125=15,94 \quad " \quad \text { " }
$$

Differenz zwischen I und II: $\mathbf{0 , 0 1 5}$ Proc. Hb.

Die Mitte des Glaskeils unseres Apparates zeichnet sich also

\begin{tabular}{|c|c|c|c|c|c|c|c|c|}
\hline $\mathrm{Nr}$. & \multicolumn{2}{|l|}{ Schichtdicke $15 \mathrm{~mm}$} & \multicolumn{3}{|c|}{ Sehichtdicke $12 \mathrm{~mm}$} & \multicolumn{3}{|c|}{ Differenz } \\
\hline 1 & Theilstr. $25,00=6,147 \mathrm{P}$ & roc. $\mathrm{Hb}$ & Theil & $19,95=6,134 \mathrm{P}$ & roc.Hb & 0,013 & Proc & $\mathrm{Hb}$ \\
\hline 2 & $=41,1=11,60$ & $=$ & $=$ & $36,8=11,57$ & $=$ & 0,03 & $=$ & $=$ \\
\hline 3 & $69,30=17,31$ & $=$ & $=$ & $55,30=17,26$ & $=$ & 0,05 & $=$ & $=$ \\
\hline 4 & $95,80=15,966$ & $=$ & $=$ & $76,60=15,958$ & $=$ & 0,006 & $=$ & $=$ \\
\hline
\end{tabular}
durch grosse Genauigkeit für wechselnde Sehichtdicke und Verdiunnungen aus. Dasselbe erweisen für die oberen und unteren Regionen die folgenden Beispiele, welche den Versuchsprotocollen entnommen sind. Für jede Schichtdicke sind 10 Ablesungen gemacht.

Es muss also das Instrument unseres Institutes als ein für vergleichende Untersuchung relativer $\mathrm{Hb}$-Werthe (und nur auf solche kommt es für diese Untersuchungen an) ganz besonders genaues bezeichnet werden.

Die absolute Aichung ist Mangels eines Spectrophotometers nicht vorgenommen, da ich bislang das Instrument für absolute $\mathrm{Hb}-\mathrm{Be}-$

\begin{tabular}{|c|c|c|c|c|c|c|}
\hline \multicolumn{2}{|c|}{$\begin{array}{l}\text { Spectralapparat } \\
\text { (Kríss) }\end{array}$} & \multicolumn{2}{|c|}{$\begin{array}{l}\text { Hämoglobinometer } \\
\text { (Petroleumlicht) }\end{array}$} & \multicolumn{3}{|c|}{ Differenz } \\
\hline \multicolumn{2}{|c|}{0,480 Proc. $\mathrm{Hb}$} & \multicolumn{2}{|c|}{ 0,484 Proc.Hb } & \multicolumn{3}{|c|}{$+0,004$ Proe. $\mathrm{Hb}$} \\
\hline 0,393 & $=\quad=$ & 0,396 & & $+0,003$ & $=$ & $=$ \\
\hline 0,488 & $=$ & 0,467 & $=$ & $-0,021$ & $=$ & $=$ \\
\hline 0,606 & $=$ & 0,590 & $=$ & $-0,016$ & $=$ & $=$ \\
\hline 0,582 & $=$ & 0,608 & & $+0,026$ & $=$ & $=$ \\
\hline 0,656 & $=$ & 0,678 & $=$ & $+0,022$ & $=$ & $=$ \\
\hline 0,701 & $=$ & 0,722 & $=$ & $+0,021$ & $=$ & $=$ \\
\hline 0,536 & $=$ & 0,528 & $=$ & $-0,008$ & $=$ & $=$ \\
\hline 0,577 & $=$ & 0,572 & $=$ & $-0,005$ & $=$ & $=$ \\
\hline 0,574 & $==$ & 0,572 & $=$ & $-0,002$ & $=$ & $=$ \\
\hline
\end{tabular}
stimmungen nicht benutzt habe, doch verdanke ich der Liebenswïrdigkeit von Herrn Prof. Hans Meyer in Marburg eine grössere Reihe von Vergleichsbestimmungen des Hämoglobinometers mit dem Krïss'schen Spectralapparat, der ich folgende Zahlen entnehme: 
Für die freundliche Ueberlassung dieser Zahlen sprechen wir auch an dieser Stelle unseren besten Dank aus. Berücksichtigt man, dass dabei für die Hämoglobinometerbestimmung jeweils nur $4-6$ Ablesungen gemacht wurden, so ist die Uebereinstimmung eine durohaus befriedigende. Die Differenzen sind abwechselnd positiv und negativ. - Wenn man demnach die an diesen beiden Instrumenten von verschiedenen Beobachtern gemaehten Erfahrungen vergleicht, so muss der Miescher'sehe Apparat als ein zuverlässiges Instrument bezeichnet werden. -

Analysenwerthe der Hundeversuche I-VI.

A. Im Blut resp. Serum:

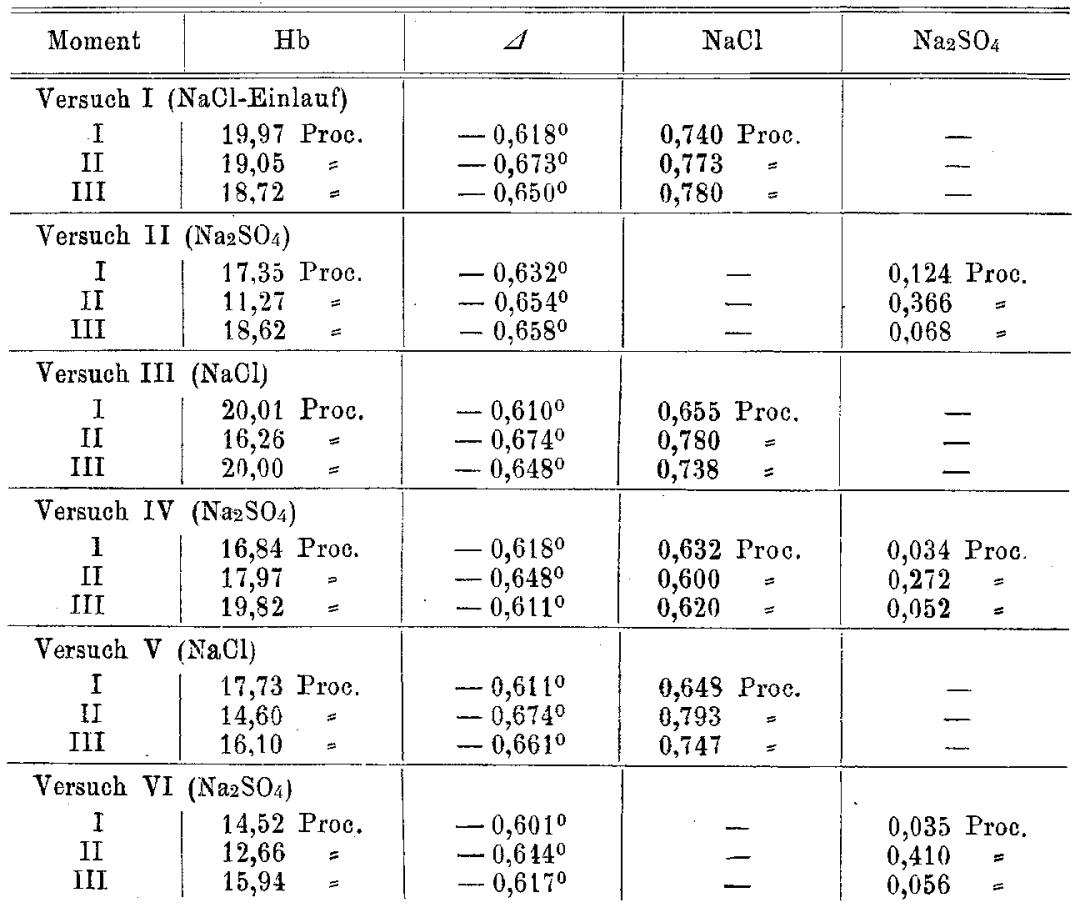

B. Im Harn :

\begin{tabular}{|c|c|c|c|c|c|c|c|c|c|c|c|c|}
\hline Versuch & \multicolumn{2}{|c|}{ I } & \multicolumn{2}{|c|}{ II } & \multicolumn{2}{|c|}{ ITI } & \multicolumn{2}{|c|}{ IV } & \multicolumn{2}{|c|}{ V } & \multicolumn{2}{|c|}{$\mathrm{VI}$} \\
\hline $\begin{array}{l}\text { Harn- } \\
\text { portion }\end{array}$ & I & $1 I$ & I & II & I & II & I & I1 & I & II & I & II \\
\hline $\mathrm{cem}$ & 37,4 & 27,9 & 66,9 & 46,4 & 65,4 & 88,0 & 105,0 & 119,7 & 17,2 & 57,8 & 93,3 & 90,9 \\
\hline$\% \mathrm{NaCl}$ & 1,288 & 1,627 & - & - & 1,182 & 1,220 & 0,067 & 0,050 & 1,080 & 0,822 & 0,067 & 0,073 \\
\hline$\% / 0 \mathrm{Na}_{2} \mathrm{SO}_{4}$ & - & - & 2,915 & 3,873 & - & - & 2,159 & 3,124 & - & - & 2,454 & 3,562 \\
\hline
\end{tabular}

